\title{
About Controlling of Regimes of Heating During Growth a Heterostructures from Gas Phase
}

\author{
E.L. Pankratov ${ }^{1, *}$, E.A. Bulaeva ${ }^{2}$ \\ ${ }^{1}$ Nizhny Novgorod State University, 23 Gagarin avenue, Nizhny Novgorod, 603950, Russia \\ ${ }^{2}$ Nizhny Novgorod State University of Architecture and Civil Engineering, 65 Il'insky Street, Nizhny Novgorod, 603950, Russia \\ *Corresponding Author: elp2004@mail.ru
}

Copyright $@ 2013$ Horizon Research Publishing All rights reserved.

\begin{abstract}
In this paper we analyzed distribution of temperature in a reactor for epitaxial growth from gaze phase. Based on the analysis it has been formulated recommendations to increase homogeneity of distribution of temperature in depth of heterostructure, which has been grown in the reactor.
\end{abstract}

Keywords Gas Phase Epitaxy; Analytical Approach To Model of Epitaxy Process; Optimization of Epitaxy Process

\section{Introduction}

In the present time most part of the solid state electronic devices are manufacturing based on heterostructures [1-10]. It could be used different methods to growth heterostructures: molecular-beam epitaxy, epitaxy from gas phase, magnetron sputtering [1,6]. One of problems of manufacturing of heterostructures is growth of the epitaxial layers with larger homogeneity of their properties. In this paper we consider an approach to increase the homogeneity of the properties during growth of heterostructures from gas phase. To illustrate the approach we consider a vertical reactor for epitaxy from gas phase (see Fig. 1). In the composition of the reactor it has been used keeper of substrate. A substrate could be fixed on the keeper and revolves with angular velocity $\omega$. A spiral presents around the keeper of substrate. Let us consider an electrical current in the spiral. This current gives us possibility to heat the keeper of substrate and the substrate due to electro-magnetic irradiation and scin-effect. Some materials in gas form for growth required heterostructure are inserting from the substrate in admixture with a gas-carrier. Let us consider an external casing to decrease losing of the above materials in gas phase (gases-reagents) and the gas-carrier from outside of the device. The above heating, which has been induced by electrical current in spiral, is necessary for activation of chemical reaction between materials in gas form, which are used for growth of epitaxial layers of heterostructure. It is practicably to use such regime of epitaxial growth, which corresponds to maximal increasing of homogeneity of temperature in depth of heterostructure. Using the regime attracted an interest to increase homogeneity of properties of layers of heterostructures. Main aim of the present paper is determination of conditions of growth of above heterostructure, which give us possibility to increase homogeneity of properties of the structure.

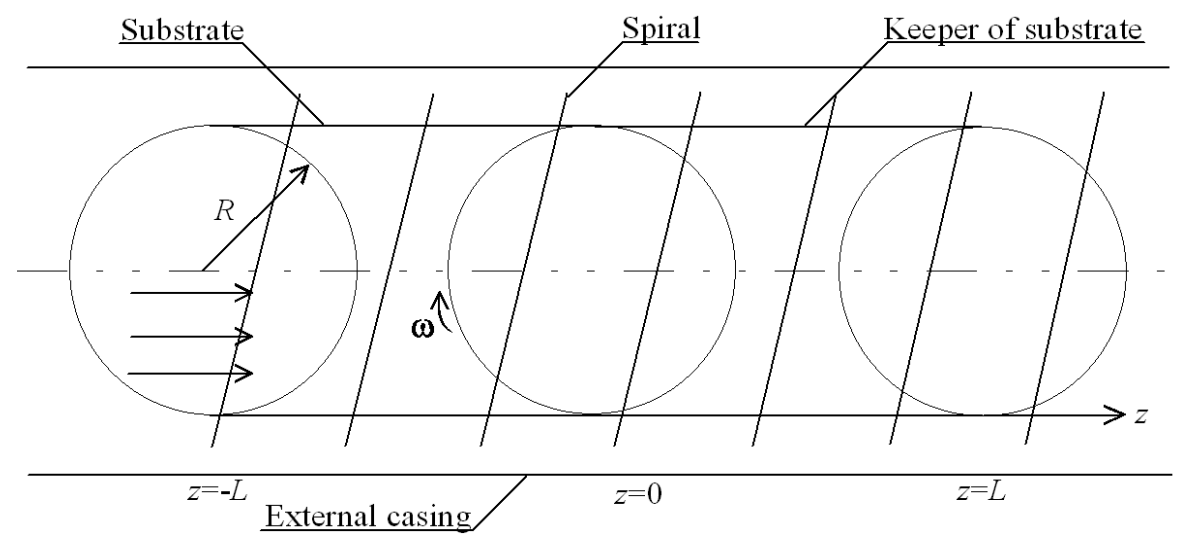

Figure 1. A plant for the gas phase epitaxy in neighborhoods of reaction zone 


\section{Method of Solution}

To solve our aims we determine spatio-temporal distribution of temperature. We determined the required distribution of temperature by solving the following boundary problem [11]

$$
c(T) \frac{\partial T(\vec{r}, t)}{\partial t}=\operatorname{div}\{\lambda \cdot \operatorname{grad}[T(\vec{r}, t)]-\vec{v}(\vec{r}, t) \cdot c(T) \cdot T(\vec{r}, t) \cdot C(\vec{r}, t)\}+p(\vec{r}, t),
$$

where $\vec{v}$ is the speed of flow of mixture of gases; $\vec{r}=\vec{r}(r, \varphi, z)$ - is the radius-vector in spherical system of coordinate; $c(T)$ is the heat capacitance; $T(\vec{r}, t)$ is the spatio-temporal distribution of temperature; $p(\vec{r}, t)$ is the density of heat power, which has been generated in the system keeper of substrate - substrate; $r, \varphi, z$ and $t$ are the current cylindrical coordinates and time, respectively; $C(\vec{r}, t)$ is the spatio-temporal distribution of concentration of mixture of gases. The first term of the Eq. (1) describes diffusion of gas from beginning of reactor $(z=-L)$ to end of the reactor $(z=L)$. The second term of the Eq. (1) describes convection trans-port of gas with velocity of flow $\vec{v}$ under influence of external pressure $P$.

To solve this boundary problem it is necessary to take into account stream of mixture of gases and concentration of the mixture. Let us determine the required values by solving the equation of Navier-Stokes and the second Fourier law. We also assume that radius of keeper of substrate $R$ essentially larger, than thickness of diffusion and near-boundary layers. We also assume, that stream of gas is laminar. In this situation the appropriate equations could be written as

$$
\begin{array}{r}
\frac{\partial \vec{v}}{\partial t}+(\vec{v} \cdot \nabla) \vec{v}=-\nabla\left(\frac{P}{\rho}\right)+v \Delta \vec{v}, \\
\frac{\partial C(\vec{r}, t)}{\partial t}=\operatorname{div}\{D \cdot \operatorname{grad}[C(\vec{r}, t)]-\vec{v}(\vec{r}, t) \cdot C(\vec{r}, t)\},
\end{array}
$$

where $D$ is the diffusion coefficient of mixture of gases (gases-reagents and gas- carrier); $P$ is the pressure; $\rho$ is the density of gases; $v$ is the kinematical viscosity. Let us consider the regime of the limiting flow, when all forthcoming to the disk molecules of deposit material are deposing on the substrate, flow is homogenous and one dimension. In this case boundary and initial conditions could be written as

$$
\begin{gathered}
C(r, \varphi,-L, t)=C_{0}(t), C(r, \varphi, 0, t)=0, C(r, 0, z, t)=C(r, 2 \pi, z, t), C(r, \varphi, z, 0)=C_{0}(t) \delta(z+L), \\
C(0, \varphi, z, t) \neq \infty,\left.\frac{\partial C(\vec{r}, t)}{\partial r}\right|_{r=R}=0,\left.\frac{\partial C(\vec{r}, t)}{\partial \phi}\right|_{\phi=0}=\left.\frac{\partial C(\vec{r}, t)}{\partial \phi}\right|_{\phi=2 \pi},\left.\frac{\partial T(\vec{r}, t)}{\partial \phi}\right|_{\phi=0}=\left.\frac{\partial T(\vec{r}, t)}{\partial \phi}\right|_{\phi=2 \pi}, \\
-\left.\lambda \frac{\partial T(\vec{r}, t)}{\partial r}\right|_{r=R}=\sigma T^{4}(R, \phi, z, t),-\left.\lambda \frac{\partial T(\vec{r}, t)}{\partial z}\right|_{z=-L}=\sigma T^{4}(r, \phi,-L, t),, T(r, \varphi, z, 0)=T_{r} \\
\left.\frac{\partial v_{r}(\vec{r}, t)}{\partial r}\right|_{r=0}=\left.\frac{\partial v_{r}(\vec{r}, t)}{\partial r}\right|_{r=R},\left.\frac{\partial v_{\phi}(\vec{r}, t)}{\partial \phi}\right|_{\phi=0}=\left.\frac{\partial v_{\phi}(\vec{r}, t)}{\partial \phi}\right|_{\phi=2 \pi}, T(r, 0, z, t)=T(r, 2 \pi, z, t), \\
\left.\frac{\partial v_{r}(\vec{r}, t)}{\partial r}\right|_{r=0}=\left.\frac{\partial v_{r}(\vec{r}, t)}{\partial r}\right|_{r=R},\left.\frac{\partial v_{\phi}(\vec{r}, t)}{\partial \phi}\right|_{\phi=0}=\left.\frac{\partial v_{\phi}(\vec{r}, t)}{\partial \phi}\right|_{\phi=2 \pi}, v_{\varphi}(r, \varphi, z, 0)=0, v_{z}(r, \varphi,-L, 0)=V_{0}(4) \\
-\left.\lambda \frac{\partial T(\vec{r}, t)}{\partial z}\right|_{z=L}=\sigma T^{4}(r, \phi, L, t), T(0, \varphi, z, t) \neq \infty, v_{r}(r, \varphi,-L, t)=0, v_{r}(r, \varphi, 0, t)=0, \\
v_{r}(r, \varphi, L, t)=0, T(r, \varphi, z, 0)=T_{r}, v_{r}(r, 0, z, t)=v_{r}(r, 2 \pi, z, t), v_{r}(0, \varphi, z, t) \neq \infty, v_{\varphi}(r, \varphi, 0, t)=\omega r, \\
v_{\varphi}(r, \varphi,-L, t)=0, v_{\varphi}(r, \varphi, L, t)=0, v_{\varphi}(r, 0, z, t)=v_{\varphi}(r, 2 \pi, z, t), v_{\varphi}(0, \varphi, z, t) \neq \infty, v_{z}(r, \varphi,-L, t)=V_{0}, \\
v_{z}(r, \varphi, 0, t)=0, v_{z}(r, \varphi, L, t)=0, v_{z}(r, 0, z, t)=v_{z}(r, 2 \pi, z, t), v_{z}(0, \varphi, z, t) \neq \infty, v_{r}(r, \varphi, z, 0)=0,
\end{gathered}
$$

where $\sigma=5,67 \cdot 10^{-8} \mathrm{~W} \cdot \mathrm{m}^{-2} \cdot \mathrm{K}^{-4}, T_{r}$ is the room temperature. The conditions describe absents of gas flow through casing of reactor at $r=R$, absence of infinite increasing of approximation of concentration of gas at $r=0$, conditions of periodicity of concentration of gas with varying of angular coordinate $\varphi$, initial condition and condition at $z=-L$ correspond to presents of 
concentration of gas on beginning of reactor in initial moment of time, reason of considered value of concentration in the end of reaction $z=L$ is necessity to decrease energy cost during growth of heterostructure.

Equations for components of velocity of flow with account cylindrical system of coordinate could be written as

$$
\left\{\begin{array}{c}
\frac{\partial v_{r}}{\partial t}+v_{r} \frac{\partial v_{r}}{\partial r}+\frac{v_{\phi}}{r} \frac{\partial v_{\phi}}{\partial \phi}+v_{z} \frac{\partial v_{z}}{\partial z}=v\left(\frac{\partial^{2} v_{r}}{\partial r^{2}}+\frac{\partial^{2} v_{r}}{\partial r \partial z}-\frac{\partial^{2} v_{r}}{\partial z^{2}}+\frac{\partial^{2} v_{z}}{\partial r \partial z}\right)-\frac{\partial}{\partial r}\left(\frac{P}{\rho}\right) \\
\frac{\partial v_{\phi}}{\partial t}+v_{r} \frac{\partial v_{r}}{\partial r}+\frac{v_{\phi}}{r} \frac{\partial v_{\phi}}{\partial \phi}+v_{z} \frac{\partial v_{z}}{\partial z}= \\
=v\left(\frac{1}{r} \frac{\partial^{2} v_{r}}{\partial r \partial \phi}+\frac{2}{r^{2}} \frac{\partial^{2} v_{\phi}}{\partial \phi^{2}}-\frac{1}{r^{2}} \frac{\partial^{2} v_{r}}{\partial \phi \partial}+\frac{\partial^{2} v_{\phi}}{\partial z^{2}}\right)-\frac{1}{r} \frac{\partial}{\partial \phi}\left(\frac{P}{\rho}\right) \\
\frac{\partial v_{z}}{\partial t}+v_{r} \frac{\partial v_{r}}{\partial r}+\frac{v_{\phi}}{r} \frac{\partial v_{\phi}}{\partial \phi}+v_{z} \frac{\partial v_{z}}{\partial z}=v\left(\frac{\partial^{2} v_{r}}{\partial z^{2}}+\frac{\partial^{2} v_{z}}{\partial r^{2}}+\frac{1}{r^{2}} \frac{\partial^{2} v_{z}}{\partial \phi^{2}}\right)-\frac{\partial}{\partial z}\left(\frac{P}{\rho}\right)
\end{array}\right.
$$

Let us transform the system of Eqs.(5) to integro-differential form to simplify solution of them. The integro-differential equations are presented in the Appendix.

We determine solution of the system of equations by using method of averaging of function corrections [12-15]. Framework the approach we replace components of velocity of flow $v_{r}, v_{\varphi}$ and $v_{z}$ on their average values $\alpha_{1 r}, \alpha_{1 \varphi}$ and $\alpha_{1 z}$, i.e. $v_{r} \rightarrow \alpha_{1 r}, v_{\varphi} \rightarrow \alpha_{1 \varphi}, v_{z} \rightarrow \alpha_{1 z}$ in right sides of the integro-differential equations. After this replacement we obtained the first-order approximations of components of velocity. The approximations are presented in the Appendix. We determine the average values $\alpha_{1 r}, \alpha_{1 \varphi}$ and $\alpha_{1 z}$ by the standard relations [12-15]

$$
\begin{gathered}
\alpha_{1 r}=\int_{0}^{\Theta} \int_{0}^{R} r \int_{0}^{2 \pi} \int_{-L}^{L} v_{1 r} d z d \phi d r d t, \alpha_{1 \phi}=\int_{0}^{\Theta} \int_{0}^{R} r \int_{0}^{2 \pi} \int_{-L}^{L} v_{1 \phi} d z d \phi d r d t, \\
\alpha_{1 z}=\int_{0}^{\Theta} \int_{0}^{R} r \int_{0}^{2 \pi} \int_{-L}^{L} v_{1 z} d z d \phi d r d t,
\end{gathered}
$$

where $\Theta$ is the continuance of stream of mixture of gases. Substitution of the above first- order approximations into the relations (6) and insignificant transformations give us possibility to obtain equations for the required average values $\alpha_{1 r}, \alpha_{1 \varphi}$ and $\alpha_{1 z}$ in the following form

$$
\left\{\begin{array}{l}
a_{1} \alpha_{1 r}^{2}+a_{2} \alpha_{1 \phi}^{2}+a_{3} \alpha_{1 z}^{2}+a_{4} \alpha_{1 r}=a_{5} \\
b_{1} \alpha_{1 r}^{2}+b_{2} \alpha_{1 \phi}^{2}+b_{3} \alpha_{1 z}^{2}+b_{4} \alpha_{1 r}+b_{5} \alpha_{1 \phi}=b_{6} \\
c_{1} \alpha_{1 r}^{2}+c_{2} \alpha_{1 \phi}^{2}+c_{3} \alpha_{1 z}^{2}+c_{4} \alpha_{1 r}+c_{5} \alpha_{1 z}=c_{6}
\end{array}\right.
$$

System of equations (7) could be solved by standard approaches [16]. Values of parameters $a_{i}, b_{i}$ and $c_{i}$ are presented in the Appendix.

The second-order approximations of components of velocity of flow could be obtained by replacement of the required functions in right sides of integro-differential forms of Eqs.(5) on the following sums $v_{r} \rightarrow \alpha_{2 r}+v_{1 r}, v_{\varphi} \rightarrow \alpha_{2 \varphi}+v_{1 \varphi}$ and $v_{z} \rightarrow \alpha_{2 z}+v_{1 z}$. The approximations are presented in the Appendix. We calculated the average values $\alpha_{2 r}, \alpha_{2 \varphi}$ and $\alpha_{2 z}$ by the standard relations [12-15]

$$
\begin{gathered}
\alpha_{2 r}=\int_{0}^{\Theta} \int_{0}^{R} r \int_{0}^{2 \pi} \int_{-L}^{L}\left(v_{2 r}-v_{1 r}\right) d z d \phi d r d t, \alpha_{2 \phi}=\int_{0}^{\Theta} \int_{0}^{R} r \int_{0}^{2 \pi} \int_{-L}^{L}\left(v_{2 \phi}-v_{1 \phi}\right) d z d \phi d r d t, \\
\alpha_{2 z}=\int_{0}^{\Theta} \int_{0}^{R} r \int_{0}^{2 \pi} \int_{-L}^{L}\left(v_{2 z}-v_{1 z}\right) d z d \phi d r d t .
\end{gathered}
$$

Substitution of the first- and the second-order approximations into relations (8) gives us possibility to obtain equations for required average values 


$$
\left\{\begin{array}{l}
A_{1} \alpha_{2 r}^{2}+A_{2} \alpha_{2 \phi}^{2}+A_{3} \alpha_{2 z}^{2}+A_{4} \alpha_{2 r}+A_{5} \alpha_{2 \phi}+A_{6} \alpha_{2 z}=A_{7} \\
B_{1} \alpha_{2 r}^{2}+B_{2} \alpha_{2 \phi}^{2}+B_{3} \alpha_{2 z}^{2}+B_{4} \alpha_{2 r}+B_{5} \alpha_{2 \phi}+B_{6} \alpha_{2 z}=B_{7} \\
C_{1} \alpha_{1 r}^{2}+C_{2} \alpha_{1 \phi}^{2}+C_{3} \alpha_{1 z}^{2}+C_{4} \alpha_{1 r}+C_{5} \alpha_{1 \phi}+C_{6} \alpha_{1 z}=C_{7}
\end{array}\right.
$$

Solution of the equations could be obtain by standard approaches [16]. Values of parameters $A_{i}, B_{i}$ and $C_{i}$ are presented in the Appendix.

In this section we obtained components of velocity of flow of mixture of materials in gas phase, which are used for growth of heterostructure, and gas-carrier in the second-order approximation framework method of averaging of function corrections. Usually the second-order approximation is enough good approximation to make qualitative analysis of obtained solution and to obtain some quantitative results [13-15].

Let us rewrite Eqs.(1) and (3) by using cylindrical system of coordinate. In this situation we assume, that heat conduction coefficient and heat capacitance weakly dependent on temperature and one can neglect by the dependence. In this situation the equation could be written as

$$
\begin{gathered}
c \frac{\partial T(\vec{r}, t)}{\partial t}=\lambda \frac{\partial^{2} T(\vec{r}, t)}{\partial r^{2}}+\lambda \frac{1}{r^{2}} \frac{\partial^{2} T(\vec{r}, t)}{\partial \phi^{2}}+\lambda \frac{\partial^{2} T(\vec{r}, t)}{\partial z^{2}}-c \cdot \frac{\partial}{\partial r}\left[v_{r}(\vec{r}, t) \cdot C(\vec{r}, t) \cdot T(\vec{r}, t)\right]- \\
-\frac{c}{r} \frac{\partial}{\partial \phi}\left[v_{\phi}(\vec{r}, t) \cdot C(\vec{r}, t) \cdot T(\vec{r}, t)\right]-c \cdot \frac{\partial}{\partial z}\left[v_{z}(\vec{r}, t) \cdot C(\vec{r}, t) \cdot T(\vec{r}, t)\right]+p(\vec{r}, t), \\
\frac{\partial C(\vec{r}, t)}{\partial t}=\frac{1}{r} \frac{\partial}{\partial r}\left[r D \frac{\partial C(\vec{r}, t)}{\partial r}\right]+\frac{1}{r^{2}} \frac{\partial}{\partial \phi}\left[D \frac{\partial C(\vec{r}, t)}{\partial \phi}\right]+\frac{\partial}{\partial z}\left[D \frac{\partial C(\vec{r}, t)}{\partial z}\right]- \\
-\frac{1}{r} \frac{\partial}{\partial r}\left[r C(\vec{r}, t) v_{r}(\vec{r}, t)\right]-\frac{1}{r} \frac{\partial}{\partial \phi}\left[C(\vec{r}, t) v_{\phi}(\vec{r}, t)\right]-\frac{\partial}{\partial z}\left[C(\vec{r}, t) v_{z}(\vec{r}, t)\right] .
\end{gathered}
$$

Farther we transform the above differential equations to the following integro- differential form

$$
\begin{gathered}
T(\vec{r}, \tau)=T(\vec{r}, \tau)+\frac{1}{4 \pi R^{2} L}\left[\Gamma_{r}+\Gamma_{\phi}+\Gamma_{z}-\Lambda_{r}-\Lambda_{\phi}-\Lambda_{z}+\int_{0}^{t} \int_{R}^{r} u^{2} p(\vec{r}, \tau) d u d \tau+\right. \\
\left.+\sigma R^{2} \int_{0}^{t} T^{4}(R, \phi, z, t) d \tau-\int_{R}^{r} u^{2} T(\vec{r}, \tau) d u+\left(r^{3}-R^{3}\right) \frac{T_{r}}{3}\right], \\
C(\vec{r}, \tau)=C(\vec{r}, \tau)+\frac{1}{4 \pi R^{2} L}\left[r \int_{0}^{t} D \frac{\partial C(\vec{r}, \tau)}{\partial r} d \tau+\frac{\partial \tilde{\Gamma}_{\phi 0}(\vec{r}, \tau)}{\partial \phi}-\int_{R}^{r} u^{2} C(\vec{r}, \tau) d u+\right. \\
+\frac{\partial \tilde{\Gamma}_{z 2}(\vec{r}, \tau)}{\partial z}-\tilde{\Gamma}_{r 1}(\vec{r}, \tau)-r^{2} \int_{0}^{t} C(\vec{r}, \tau) v_{r}(\vec{r}, \tau) d \tau-\frac{\partial \tilde{\Lambda}_{\phi 1}(\vec{r}, \tau)}{\partial \phi}-\frac{\partial \tilde{\Lambda}_{z 1}(\vec{r}, \tau)}{\partial z}+ \\
\left.+\tilde{\Lambda}_{r 1}(\vec{r}, \tau)+C_{0}(t) \delta(z+L)\right],
\end{gathered}
$$

where

$$
\begin{gathered}
\Gamma_{\rho}(\vec{r}, \tau)=\lambda \int_{0}^{t} \int_{R}^{r} u \frac{\partial^{2} T(\vec{r}, \tau)}{\partial \rho^{2}} d u d \tau, \Lambda_{\rho}(\vec{r}, \tau)=c \frac{\partial}{\partial \rho} \int_{0}^{t} \int_{R}^{r} u^{2} v_{\rho}(\vec{r}, \tau) C(\vec{r}, \tau) T(\vec{r}, \tau) d u d \tau, \\
\tilde{\Gamma}_{\rho i}(\vec{r}, \tau)=\int_{0}^{t} \int_{R}^{r} u^{i} D \frac{\partial C(\vec{r}, \tau)}{\partial \rho} d u d \tau, \tilde{\Lambda}_{\rho i}(\vec{r}, \tau)=\int_{0}^{t} \int_{R}^{r} u^{i} C(\vec{r}, \tau) v_{\rho}(\vec{r}, \tau) d u d \tau .
\end{gathered}
$$

To determine spatio-temporal distributions of temperature and concentration of mixture of gases we used method of averaging of function corrections. To determine the first-order approximations of the required functions we replace the functions in right sides of Eqs. (10a) and (11a) on their average values $\alpha_{1 T}$ and $\alpha_{1 C}$. In this situation the first-order approximations could be written in this form 


$$
\begin{gathered}
T_{1}(\vec{r}, \tau)=\alpha_{1 T}+\frac{1}{4 \pi R^{2} L}\left[2 c \alpha_{1 T} \alpha_{1 C} V_{r}(\vec{r}, \tau)-c \alpha_{1 T} \alpha_{1 C} \int_{0}^{t} r^{2} v_{r}(\vec{r}, \tau) d \tau-c \alpha_{1 T} \frac{\partial V_{\varphi}(\vec{r}, \tau)}{\partial \varphi} \times\right. \\
\left.\times \alpha_{1 C}-c \alpha_{1 T} \alpha_{1 C} \frac{\partial V_{z}(\vec{r}, \tau)}{\partial z}+\int_{0}^{t} \int_{R}^{r} u^{2} p(\vec{r}, \tau) d u d \tau+\frac{T_{r}}{3}\left(r^{3}-R^{3}\right)-\frac{\alpha_{1 T}}{3}\left(r^{3}-R^{3}\right)+\sigma R^{2} \alpha_{1 T}^{4}\right], \\
C_{1}(r, \varphi, z, t)=\alpha_{1 C}+\frac{1}{4 \pi R^{2} L}\left[\alpha_{1 C} \int_{0}^{t} \int_{R}^{r} u v_{r}(u, \varphi, z, \tau) d u d \tau-\frac{\partial}{\partial \varphi} \int_{0}^{t} \int_{R}^{r} u v_{\varphi}(u, \varphi, z, \tau) d u d \tau \times\right. \\
\times \alpha_{1 C}-\alpha_{1 C} \frac{\partial}{\partial z} \int_{0}^{t} \int_{R}^{r} u^{2} v_{z}(u, \varphi, z, \tau) d u d \tau-\frac{\alpha}{3}\left(r^{3}-R^{3}\right)-r^{2} \alpha_{1 C} \int_{0}^{t} v_{r}(r, \varphi, z, \tau) d \tau+C_{0}(t) \times \\
\times \delta(z+L)] .
\end{gathered}
$$

where $V_{r}(\vec{r}, \tau)=\int_{0}^{t} \int_{R}^{r} u v_{r}(\vec{r}, \tau) d u d \tau$

We determine the average values $\alpha_{1 T}$ and $\alpha_{1 C}$ by standard relations, which are analogous to relations (6)

$$
\alpha_{1 T}=\int_{0}^{\Theta} \int_{0}^{R} r \int_{0}^{2 \pi} \int_{-L}^{L} T_{1} d z d \varphi d r d t, \alpha_{1 C}=\int_{0}^{\Theta} \int_{0}^{R} r \int_{0}^{2 \pi} \int_{-L}^{L} C_{1} d z d \varphi d r d t
$$

Substitution of the first- and the second-order approximations of concentrations and temperature into relation (12) gives us possibility to obtain the following result

$$
\alpha_{1 T}=\sqrt{\frac{y}{2}}+\frac{1}{2} \sqrt{2 y+\frac{4}{\sqrt{8 y}}(y-E)} \quad \alpha_{1 C} G+\pi R^{2} \int_{0}^{\Theta} C_{0}(t) d t=0
$$

where $E=\frac{4}{5} \pi L \Theta R^{5}-2 c \int_{0}^{\Theta}(\Theta-t) \int_{0}^{R} r^{3} \int_{0}^{2 \pi} \int_{-L}^{L} v_{r}(\vec{r}, t) d z d \varphi d r d t-c \pi V_{0} \Theta R^{2}, F=\frac{2}{5} \pi L \times$

$\times T_{r} R^{5} \Theta^{2}+\frac{1}{2} \int_{0}^{\Theta} \int_{0}^{R} r^{4} \int_{0}^{2 \pi} \int_{-L}^{L} p(\vec{r}, t) d z d \varphi d r d t, y=\sqrt[3]{\sqrt{\frac{F^{2}}{256}+\frac{E^{3}}{13824}}-\frac{F}{16}}-\sqrt[3]{\sqrt{\frac{F^{2}}{256}+\frac{E^{3}}{13824}}+\frac{F}{16}}$,

$G=\frac{1}{2} \int_{0}^{\Theta}(\Theta-t) \int_{0}^{R} r^{3} \int_{0}^{2 \pi} \int_{-L}^{L} v_{1 z}(\vec{r}, t) d z d \varphi d r d t-\frac{1}{2} \int_{0}^{\Theta}(\Theta-t) \int_{0}^{R} r^{3} \int_{0}^{2 \pi} \int_{-L}^{L} v_{r}(\vec{r}, t) d z d \varphi d r d t+$

$+3 \pi L R^{5} \frac{\Theta}{5}$.

The second-order approximations of temperature and concentration of mixture of gases we determine framework standard procedure of method of averaging of function corrections [12-15], i.e. by replacement of the required functions in the right sides of Eqs. (10a) and (11a) on the following sums $T \rightarrow \alpha_{2 T}+T_{1}, C \rightarrow \alpha_{2 C}+T_{1 T}$. In this situation the second-order approximations of required functions could be written as

$$
\begin{aligned}
& T_{2}(\vec{r}, t)=\alpha_{2 T}+T_{1}(\vec{r}, \tau)+\frac{1}{4 \pi R^{2} L}\left\{\lambda r^{2} \int_{0}^{t} \frac{\partial T_{1}(\vec{r}, \tau)}{\partial r} d \tau-2 \lambda W_{r 11}(\vec{r}, t)+\lambda W_{\varphi 01}(\vec{r}, t)+\right. \\
& +\lambda W_{z 21}(\vec{r}, t)-c \cdot r^{2} \int_{0}^{t}\left[\alpha_{2 C}+C_{1}(\vec{r}, \tau)\right]\left[\alpha_{2 T}+T_{1}(\vec{r}, \tau)\right] v_{r}(\vec{r}, \tau) d \tau+2 c \Phi_{r 01}(\vec{r}, t)-
\end{aligned}
$$




$$
\begin{gathered}
-c \Phi_{\varphi 11}(\vec{r}, t)-c \Phi_{z 12}+\int_{0}^{t} \int_{R}^{r} u^{2} p(\vec{r}, \tau) d u d \tau+\sigma R^{2} \int_{0}^{t}\left[\alpha_{2 T}+T_{1}(\vec{r}, \tau)\right]^{4} d \tau+\left(r^{3}-R^{3}\right) \frac{T_{r}}{3}- \\
\left.-\int_{R}^{r} u^{2}\left[\alpha_{2 T}+T_{1}(\vec{r}, \tau)\right] d u\right\} \\
C_{2}(\vec{r}, \tau)=C_{1}(\vec{r}, \tau)+\frac{1}{4 \pi R^{2} L}\left\{\frac{\partial \widetilde{W}_{\varphi 01}(\vec{r}, t)}{\partial \varphi}-\widetilde{W}_{r 11}(\vec{r}, t)+\frac{\partial \widetilde{W}_{z 11}(\vec{r}, t)}{\partial z}+\widetilde{\Phi}_{r 01}(\vec{r}, t)-\right. \\
-\widetilde{\Phi}_{\varphi 11}(\vec{r}, t)-\widetilde{\Phi}_{z 12}(\vec{r}, t)+r^{2} \int_{0}^{t} D \frac{\partial C_{1}(\vec{r}, \tau)}{\partial r} d \tau-r^{2} \int_{0}^{t}\left[\alpha_{2 C}+C_{1}(\vec{r}, \tau)\right] v_{r}(\vec{r}, \tau) d \tau+ \\
\left.+C_{0}(t) \delta(z+L)-\int_{R}^{r} u^{2}\left[\alpha_{2 C}+C_{1}(\vec{r}, \tau)\right] d u\right\}+\alpha_{2 C}
\end{gathered}
$$

where $W_{\rho i j}(\vec{r}, t)=\int_{0}^{t} \int_{R}^{r} u^{i} \frac{\partial T_{j}(\vec{r}, \tau)}{\partial \rho} d u d \tau, \Phi_{\rho i j}(\vec{r}, t)=\frac{\partial^{i}}{\partial \rho^{i}} \int_{0}^{t} \int_{R}^{r} u^{j} v_{\rho}(\vec{r}, \tau)\left[\alpha_{2 T}+T_{1}(\vec{r}, \tau)\right] \times$ $\times\left[\alpha_{2 C}+C_{1}(\vec{r}, \tau)\right] d u d \tau, \quad \widetilde{\Phi}_{\rho i j}(\vec{r}, t)=\frac{\partial^{i}}{\partial \rho^{i}} \int_{0 R}^{t} \int_{R}^{r} u\left[\alpha_{2 C}+C_{1}(\vec{r}, \tau)\right] v_{\rho}(\vec{r}, \tau) d u d \tau, \quad \widetilde{W}_{\rho i j}(\vec{r}, t)=$ $=\int_{0}^{t} \int_{R}^{r} u^{i} D \frac{\partial C_{1}(\vec{r}, \tau)}{\partial \rho} d u d \tau$.

We determine average values of the second-order approximations of temperature and concentration of mixture $\alpha_{2 T}$ and $\alpha_{2 C}$ by using the following standard relation

$$
\alpha_{2 T}=\int_{0}^{\Theta R} \int_{0}^{2} r \int_{0}^{2 \pi} \int_{-L}^{L}\left(T_{2}-T_{1}\right) d z d \varphi d r d t, \alpha_{2 C}=\int_{0}^{\Theta R} \int_{0}^{R} r \int_{0}^{2 \pi} \int_{-L}^{L}\left(C_{2}-C_{1}\right) d z d \varphi d r d t
$$

Substitution of the first- and the second- order approximations of temperature and concentration into relations (13) gives us possibility to obtain equations for required average values in the following form

$$
\begin{gathered}
-\frac{\lambda}{2} G_{r 311}+\lambda G_{\varphi 212}+\lambda G_{z 412}-c \int_{0}^{\Theta}(\Theta-t) \int_{0}^{R} \int_{0}^{2 \pi} \int_{-L}^{L}\left[\alpha_{2 C}+C_{1}(r, \varphi, z, t)\right]\left[\alpha_{2 T}+T_{1}(r, \varphi, z, t)\right] \times \\
\times v_{r}(r, \varphi, z, t) d z d \varphi r^{2} d r d t+\sigma V_{0} \frac{c}{2} \int_{0}^{\Theta}(\Theta-t) \int_{0}^{R} r^{4} \int_{0}^{2 \pi} \int_{-L}^{L}\left[\alpha_{2 T}+T_{1}(r, \varphi, z, t)\right]^{4} d z d \varphi d r \times \\
\times C_{0}(t) d t+2 \pi L \Theta R^{5} \frac{T_{r}}{5}+\frac{1}{2} \int_{0}^{\Theta}(\Theta-t) \int_{0}^{R} r^{4} \int_{0}^{2 \pi} \int_{-L}^{L} p(r, \varphi, z, t) d z d \varphi d r d t+R^{2} \int_{0}^{\Theta}(\Theta-t) \times \\
\times \sigma \int_{0}^{R} r \int_{0}^{2 \pi} \int_{-L}^{L}\left[\alpha_{2 T}+T_{1}(r, \varphi, z, t)\right]^{4} d z d \varphi d r d t+\frac{1}{2} \int_{0}^{\Theta} \int_{0}^{R 2 \pi} \int_{0}^{L} \int_{-L}^{L}\left[\alpha_{2 T}+T_{1}(r, \varphi, z, t)\right] d z d \varphi \times \\
\times r^{4} d r d t=0 \\
-\frac{1}{2} \int_{0}^{\Theta}(\Theta-t) \int_{0}^{R} r^{3} \int_{0}^{2 \pi} \int_{-L}^{L}\left[\alpha_{2 C}+C_{1}(r, \phi, z, t)\right] v_{r}(r, \phi, z, t) d z d \phi d r d t+\frac{1}{2} \int_{0}^{\Theta}(\Theta-t) \int_{0}^{R} r^{3} \int_{0}^{2 \pi} \int_{-L}^{L} D \times
\end{gathered}
$$




$$
\begin{gathered}
\times \frac{\partial C_{1}(r, \phi, z, t)}{\partial r} d z d \phi d r d t+\frac{1}{2} \int_{0}^{\Theta} \int_{0}^{R} r^{4} \int_{0}^{2 \pi} \int_{-L}^{L}\left[\alpha_{2 C}+C_{1}(r, \phi, z, t)\right] d z d \phi d r d t+\int_{0}^{\Theta}(\Theta-t) \times \\
\times \frac{V_{0}}{2} \int_{0}^{R} r^{4} \int_{0}^{2 \pi} \int_{-L}^{L}\left[\alpha_{2 C}+C_{0}(t)\right] d z d \phi d r d t+\pi R^{5} L \int_{0}^{\Theta} C_{0}(t) d t-\int_{0}^{\Theta}(\Theta-t) \int_{0}^{R} \int_{0}^{2 \pi} \int_{-L}^{L} v_{r}(r, \phi, z, \tau) \times \\
\times\left[\alpha_{2 C}+C_{1}(r, \phi, z, \tau)\right] d z d \phi r^{3} d r d t=0 .
\end{gathered}
$$

where $G_{\rho i j k}=\int_{0}^{\Theta}(\Theta-t) \int_{0}^{R} r^{i} \int_{0}^{2 \pi} \int_{-L}^{L} \frac{\partial^{k} T_{j}(r, \phi, z, t)}{\partial \rho^{k}} d z d \phi d r d t$.

The obtained system of equations for the average values $\alpha_{2 T}$ and $\alpha_{2 C}$ could be solved by standard approaches [16]. However the obtained solution is bulky due to nonlinear dependence of diffusion coefficient on temperature. In this situation we will not present the solution in this work.

\section{Discussion}

In this section based on calculated in previous section relations for spatio-temporal distributions of temperature, components of velocity of flow of mixture of gases and their concentration we analyzed dynamics of temperature in keeper of substrate and in heterostructure grown. During initial stage one can find heating of keeper of substrate and of heterostructure grown. Time of heating could be estimated by approach, described in [16]. Value of this time is approximately equal to $\vartheta \approx(6 \pi-1) \mathrm{R} 2 / 24 \mathrm{D} 0$. Farther one can find stationary regime. After that heating of the considered system will be continued due to heating of scin-layer of keeper of substrate and of heterostructure grown. At the same time one can losing of heat due to convective heat transfer and thermal radiation. To obtain heterostructure with as much as possible homogeneity of it's properties it is practicably to make distribution of temperature with as much as possible homogeneity in stationary regime of growth. At the same time one can obtain increasing of homogeneity of distribution of temperature in keeper of substrate. To increase the homogeneity the obtaining by heterostructure heat should compensate losing of heat due to convective heat transfer and thermal radiation. In this situation it should be performed the following condition: $\int_{0}^{R} r \cdot p(r, \phi, z, t) d r \approx \sigma \cdot T^{4}(R, \phi, z, t)+\Theta \cdot v_{z}(R, \phi, z, t) / 4 \pi L R^{2}$.

\section{Conclusion}

In this paper we introduce an approach to increase homogeneity of distribution of temperatures in their depth during grown from gas phase. Increasing of homogeneity of temperature in depth of heterostructure gives us possibility to increase homogeneity of properties of appropriate materials of heterostructure grown. At the same time we introduce an analytical approach to model technological process: distribution of temperature, mixture of gases (materials for growth of heterostructure in gas phase and gas-carrier) and components of velocity of mixture of gases in vertical reactor from gas phase.

\section{Acknowledgments}

This work is supported by the contract 11.G34.31.0066 of the Russian Federation Government and educational fellowship for scientific research.

\section{Appendix}

Integral form of equations for components of velocity of flow of composition of gases (gas-carrier and materials in gas phase, which are used for formation epitaxial layer) $v_{r}, v_{\varphi}$ and $v_{z}$ could be written as

$$
v_{r}=v_{r}+\frac{v}{2 R L}\left\{S_{r r 010100}(\vec{r}, t)+S_{r r 000100}(\vec{r}, t)+S_{r z 000100}(\vec{r}, t)-S_{z r 0000001}(\vec{r}, t)-\right.
$$


$-S_{t r 1011100}(\vec{r}, t)-S_{t r 0011210}(\vec{r}, t)-\frac{1}{2} S_{t r 0012100}(\vec{r}, t)-\frac{1}{2} S_{r r 1012210}(\vec{r}, t)-\frac{1}{2} S_{r z 1002100}(\vec{r}, t)-$ $-S_{\varphi \varphi 1012210}(\vec{r}, t)+\left.r S_{r r 0011100}(\vec{r}, t)\right|_{r=R}-\left.S_{r r 0001000}(\vec{r}, t)\right|_{r=R}+\left.r S_{t r 0011210}(\vec{r}, t)\right|_{r=R}+r \times$ $\times S_{z r 0001000}(\vec{r}, t)+\left.\frac{r}{2} S_{r r 0012210}(\vec{r}, t)\right|_{r=R}-\left.S_{r r 1001000}(\vec{r}, t)\right|_{r=R}-S_{r z 0001000}(\vec{r}, t)+\frac{r}{2} S_{z z 0012210}(\vec{r}, t)-$ $-S_{r r 1011100}(\vec{r}, t)+\frac{r}{2} S_{\varphi \varphi 1002210}(\vec{r}, t)-S_{t r 1011210}(\vec{r}, t)-\frac{1}{2} S_{r r 1012100}(\vec{r}, t)-\frac{1}{2} S_{r \varphi 0012210}(\vec{r}, t)-$ $-\frac{1}{2} S_{r z 0002100}(\vec{r}, t)-\frac{1}{2} S_{z z 0002210}(\vec{r}, t)+\left.\frac{1}{2} S_{r r 0012100}(\vec{r}, t)\right|_{r=R}-R S_{r r 0001000}(\vec{r}, t)-\left[-\left.S_{r r 1011100}(\vec{r}, t)\right|_{z=-L}-\right.$ $-\left.S_{t r 0011210}(\vec{r}, t)\right|_{z=-L}-\left.\frac{1}{2} S_{r r 1012210}(\vec{r}, t)\right|_{z=-L}-\left.S_{r r 0001000}(\vec{r}, t)\right|_{z=-L}-\left.\frac{1}{2} S_{r r 0012100}(\vec{r}, t)\right|_{z=-L}-$ $+\left.\frac{1}{2} S_{\varphi \varphi 1012210}(\vec{r}, t)\right|_{z=-L}+\left.S_{z z 0001000}(\vec{r}, t)\right|_{z=-L}+\left.\frac{1}{2} S_{\varphi \varphi 0012210}(\vec{r}, t)\right|_{z=-L}-\left.r S_{t r 0011210}(\vec{r}, t)\right|_{\substack{r=R \\ z=-L}}+$ $-\left.\frac{1}{2} S_{\varphi \varphi 0012210}(\vec{r}, t)\right|_{\substack{r=R \\ z=-L}}+\left.r S_{t r 0011210}(\vec{r}, t)\right|_{\substack{r=R \\ z=-L}}+\left.r S_{r r 0011100}(\vec{r}, t)\right|_{\substack{r=R \\ z=-L}}+\left.S_{r r 0010001}(\vec{r}, t)\right|_{\substack{r=R \\ z=-L}}+\frac{r}{2} \times$ $\times\left. S_{r r 0012210}(\vec{r}, t)\right|_{\substack{r=R \\ z=-L}}+\left.S_{r r 0001000}(\vec{r}, t)\right|_{\substack{r=R \\ z=-L}}-\left.\frac{V_{0}^{2}}{2} S_{r r 0000100}(\vec{r}, t)\right|_{\substack{r=R \\ z=-L}}+\frac{1}{2} S_{r r 0012100}(\vec{r}, t)_{\substack{r=R \\ z=-L}}+$ $+\left.S_{r r 0012100}(\vec{r}, t)\right|_{\substack{r=R \\ z=-L}}+\left.\frac{r}{2} S_{\varphi \varphi 0002210}(\vec{r}, t)\right|_{\substack{r=R \\ z=-L}}+\left.\frac{r}{2} S_{z z 0012210}(\vec{r}, t)\right|_{\substack{r=R \\ z=-L}}-\left.S_{t \varphi 1010011}(\vec{r}, t)\right|_{\substack{r=R \\ z=-L}}+$ $+\left.\frac{1}{2} S_{r r 0012100}(\vec{r}, t)\right|_{\substack{r=R \\ z=-L}}-\left.S_{t r 1011210}(\vec{r}, t)\right|_{\substack{r=R \\ z=-L}}+\left.S_{z z 0001000}(\vec{r}, t)\right|_{\substack{r=R \\ z=-L}}+\left.\frac{1}{2} S_{z z 0002100}(\vec{r}, t)\right|_{\substack{r=R \\ z=-L}}-$ $\left.-\left.\frac{1}{2} S_{\varphi \varphi 0012210}(\vec{r}, t)\right|_{\substack{r=R \\ z=-L}}+\frac{1}{2} S_{z z 0002210}(\vec{r}, t)_{\substack{r=R \\ z=-L}}-\left.\frac{1}{2} S_{r r 1012210}(\vec{r}, t)\right|_{\substack{r=R \\ z=-L}}+\int_{0}^{t \varphi} \int_{0}^{t} \int_{-L}^{0} \frac{P}{\rho} d w d v d \tau\right] \frac{z}{L}-$ $\left.-\int_{0}^{t} \int_{0}^{r} \int_{0}^{\varphi z} \frac{P}{\rho} d w d v d \tau+\int_{0}^{t} \int_{0}^{r} \int_{0}^{\varphi} \int_{0}^{\varphi z} \frac{P}{\rho} d w d v d u d \tau\right\}$ $v_{\varphi}=v_{\varphi}+\frac{v}{2 L R}\left(S_{r r 0011000}(\vec{r}, t)-r S_{r r 0010001}(\vec{r}, t)-S_{z \varphi 2100001}(\vec{r}, t)+S_{r r 1001000}(\vec{r}, t)+S_{r \varphi 2111100}(\vec{r}, t)+\right.$ $+\frac{r^{2}}{2} S_{r r 0111101}(\vec{r}, t)+S_{t \varphi 2111210}(\vec{r}, t)-S_{r r 2112100}(\vec{r}, t)-S_{r r 2112100}(\vec{r}, t)+\frac{1}{2} S_{r r 2112210}(\vec{r}, t)+$ $+\frac{1}{2} S_{z z 2102100}(\vec{r}, t)+\frac{1}{2} S_{\varphi \varphi 1012100}(\vec{r}, t)+\frac{1}{2} S_{z z 2112210 l m n o}(\vec{r}, t)+\frac{\varphi}{2 \pi}\left[\left.r S_{r r 0010001}(\vec{r}, t)\right|_{z=2 \pi}-\right.$ 


$$
\begin{aligned}
& -\left.\frac{r^{2}}{2} S_{r r 0111101}(\vec{r}, t)\right|_{z=2 \pi}-\left.S_{r r 0010001}(\vec{r}, t)\right|_{z=2 \pi}-\left.S_{r r 0001000}(\vec{r}, t)\right|_{z=2 \pi}-\left.S_{t \varphi 2110011}(\vec{r}, t)\right|_{z=2 \pi}- \\
& -\left.S_{t \varphi 2111210}(\vec{r}, t)\right|_{z=2 \pi}+\left.S_{z \varphi 1100001}(\vec{r}, t)\right|_{z=2 \pi}+\left.S_{r r 2112100}(\vec{r}, t)\right|_{z=2 \pi}-\left.\frac{1}{2} S_{r r 2112210}(\vec{r}, t)\right|_{z=2 \pi}- \\
& \left.-\left.\frac{1}{2} S_{z z 2102100}(\vec{r}, t)\right|_{z=2 \pi}-\left.\frac{1}{2} S_{z z 2112210}(\vec{r}, t)\right|_{z=2 \pi}-\left.\frac{1}{2} S_{\varphi \varphi 1012100}(\vec{r}, t)\right|_{z=2 \pi}\right]-(2 \pi-\varphi) \omega^{2} r^{4} t \frac{\varphi}{4}+ \\
& +\frac{z}{L}\left\{\left.r^{2} S_{r r 0010001}(\vec{r}, t)\right|_{z=L}-\left.r S_{r r 0011000}(\vec{r}, t)\right|_{z=L}-\left.S_{r r 0011000}(\vec{r}, t)\right|_{z=L}-\left.\frac{r^{2}}{2} S_{z r 0111101}(\vec{r}, t)\right|_{z=L}-\right. \\
& -\left.S_{t \varphi 2110101}(\vec{r}, t)\right|_{z=L}-\left.S_{t \varphi 2111210}(\vec{r}, t)\right|_{z=L}-\left.\frac{1}{2} S_{r r 2112210}(\vec{r}, t)\right|_{z=L}-\left.S_{r r 2112100}(\vec{r}, t)\right|_{z=L}- \\
& -\left.S_{r r 0001000}(\vec{r}, t)\right|_{z=L}-\left.\frac{1}{2} S_{z z 2112210}(\vec{r}, t)\right|_{z=L}-\left.\frac{1}{2} S_{\varphi \varphi 1012100}(\vec{r}, t)\right|_{z=L}-\frac{1}{2} S_{z z 2102100}(\vec{r}, t)- \\
& -\left|r S_{r r 0010001}(\vec{r}, t)\right|_{\varphi=2 \pi}-\left.S_{r r 0011000}(\vec{r}, t)\right|_{\varphi=2 \pi}-\left.r^{2} S_{r r 0111101}(\vec{r}, t)\right|_{\varphi=2 \pi}-\left.S_{r r 0001000}(\vec{r}, t)\right|_{\varphi=2 \pi}- \\
& -\left.S_{t \varphi 2111210}(\vec{r}, t)\right|_{z=L}-\left.S_{t \varphi 2110101}(\vec{r}, t)\right|_{z=L}-\left.\frac{1}{2} S_{r r 2112210}(\vec{r}, t)\right|_{\substack{z=L \\
\varphi=2 \pi}}+\left.S_{r r 2112100}(\vec{r}, t)\right|_{\substack{z=L \\
\varphi=2 \pi}}- \\
& \left.\left.\left.-\left.\frac{1}{2} S_{r z 2102100}(\vec{r}, t)\right|_{\substack{z=L \\
\varphi=2 \pi}}-\left.\frac{1}{2} S_{z z 2102210}(\vec{r}, t)\right|_{\substack{z=L \\
\varphi=2 \pi}}\right] \frac{\varphi}{2 \pi}+(2 \pi-\varphi) \omega^{2} r^{4} t \frac{\varphi}{4}\right\}-\int_{0}^{t} \iint_{0}^{r} \frac{P}{\rho} d w d u d \tau\right) \\
& v_{z}=\frac{v}{2 R L}\left\{S_{z r 1100001}(\vec{r}, t)+S_{r z 0110001}(\vec{r}, t)+S_{z z 1011000}(\vec{r}, t)-\frac{1}{2} S_{r r 1112210}(\vec{r}, t)-\frac{1}{2} S_{r r 1112100}(\vec{r}, t)-\right. \\
& -S_{t z 1111210}(\vec{r}, t)-\frac{1}{2} S_{\varphi \varphi 0112210}(\vec{r}, t)-\frac{1}{2} S_{z z 1112210}(\vec{r}, t)-S_{z z 1111100}(\vec{r}, t)-\frac{1}{2} S_{z z 1112100}(\vec{r}, t)- \\
& -\frac{1}{2} S_{\varphi \varphi 0112100}(\vec{r}, t)-\frac{z}{L}\left[\left.r S_{r z 0110001}(\vec{r}, t)\right|_{z=-L}-\left.S_{t \varphi 1110101}(\vec{r}, t)\right|_{z=-L}-\left.S_{t z 1110211}(\vec{r}, t)\right|_{z=-L}+r^{2} \times\right. \\
& \times\left. S_{r r 0111101}(\vec{r}, t)\right|_{z=-L}+\left.\frac{1}{2} S_{r r 1112210}(\vec{r}, t)\right|_{z=-L}+\left.S_{\varphi z 1010001}(\vec{r}, t)\right|_{z=-L}-\left.\frac{1}{2} S_{\varphi \varphi 1012100}(\vec{r}, t)\right|_{z=-L}- \\
& \left.-\left.\frac{1}{2} S_{z z 1102100}(\vec{r}, t)\right|_{z=-L}-\left.\frac{1}{2} S_{z z 1112210}(\vec{r}, t)\right|_{z=-L}-\left.\frac{L}{2} S_{z z 1102210}(\vec{r}, t)\right|_{z=-L}-\left.\frac{1}{2} S_{\varphi \varphi 1012210}(\vec{r}, t)\right|_{z=-L}\right]- \\
& \left.-\int_{0}^{t} \int_{0}^{r}(r-u) \int_{0}^{\varphi}(\varphi-v) \frac{P}{\rho} d v d u d \tau\right\}+v_{z} \\
& \text { where } S_{\rho \gamma i j k l m n o}(\vec{r}, t)=\int_{0}^{t} \int_{0}^{r}(r-u)^{i} \int_{0}^{\varphi}(\varphi-v)^{j} \int_{0}^{z}(z-w)^{k} \frac{v_{\gamma}^{l}}{v^{m}}\left(\frac{\partial v}{\partial \rho}\right)^{n}\left(\frac{\partial v_{\gamma}}{\partial \rho}\right)^{o} d w d v d u d \tau \text {. }
\end{aligned}
$$


The first-order approximations of components of velocity of flow of composition of gases (gas-carrier and materials in gas phase, which are used for formation epitaxial layer) $\mathrm{vr}, \mathrm{v} \varphi$ and $\mathrm{vz}$ could be written as

$$
\begin{aligned}
& v_{1 r}=\alpha_{1 r}+\frac{v}{2 L R}\left\{\alpha_{1 r} \varphi^{2} R z \frac{t}{2}+\alpha_{1 r} \operatorname{tr} \varphi z+\alpha_{1 z} \operatorname{tr} \varphi z-\alpha_{1 r} S_{t r 1010210}(\vec{r}, t)-\alpha_{1 r} r^{2} \varphi \frac{t}{2}-\alpha_{1 r} \times\right. \\
& \times S_{t r 1010100}(\vec{r}, t)-\frac{\alpha_{1 r}^{2}}{2} S_{r r 0010100}(\vec{r}, t)-\frac{\alpha_{1 z}^{2}}{2} S_{r r 1000100}(\vec{r}, t)-\frac{\alpha_{1 r}^{2}}{2} S_{r r 1010210}(\vec{r}, t)-S_{\varphi \varphi 1010210}(\vec{r}, t) \times \\
& \times \frac{\alpha_{1 \varphi}^{2}}{2}-\frac{\alpha_{1 z}^{2}}{2} S_{z z 1010210}(\vec{r}, t)-\alpha_{1 r} r S_{t r 0010210}(\vec{r}, t)+\alpha_{1 r} r S_{t r 0010210}(\vec{r}, t)+\alpha_{1 r}^{2} \frac{r}{2} S_{r r 0010210}(\vec{r}, t)+ \\
& +\alpha_{1 r} R \varphi(r-z) t+\left.\alpha_{1 z}^{2} \frac{r}{2} S_{z z 0110210}(\vec{r}, t)\right|_{r=R}-\alpha_{1 r} S_{t r 1010210}(\vec{r}, t)-\alpha_{1 r} R(2 R+z) \varphi t+\alpha_{1 r} \times \\
& \times\left. S_{t z 1010210}(\vec{r}, t)\right|_{r=R}+\left.\alpha_{1 \varphi}^{2} \frac{r}{2} S_{\varphi \varphi 0000210}(\vec{r}, t)\right|_{r=R}-\left.\frac{\alpha_{1 \varphi}^{2}}{2} S_{\varphi \varphi 0010210}(\vec{r}, t)\right|_{r=R}-\left.S_{r r 0000100}(\vec{r}, t)\right|_{r=R} \times \\
& \times \frac{\alpha_{1 z}^{2}}{2}-\left.\frac{\alpha_{1 r}^{2}}{2} S_{r r 1010210}(\vec{r}, t)\right|_{r=R}+\left.\frac{\alpha_{1 r}^{2}}{2} S_{r r 0010100}(\vec{r}, t)\right|_{r=R}-\left.\frac{\alpha_{1 z}^{2}}{2} S_{z z 0000210}(\vec{r}, t)\right|_{r=R}-\alpha_{1 r} t R^{2} \varphi- \\
& -\frac{z}{L}\left[\alpha_{1 r} \operatorname{tr} \varphi L-\left.\alpha_{1 r} S_{r r 1010100}(\vec{r}, t)\right|_{z=-L}-\left.\alpha_{1 r} S_{t r 1010210}(\vec{r}, t)\right|_{z=-L}-\left.\frac{\alpha_{1 r}^{2}}{2} S_{r r 1010210}(\vec{r}, t)\right|_{z=-L}-\right. \\
& -\left.\frac{\alpha_{1 \varphi}^{2}}{2} S_{\varphi \varphi 0010210}(\vec{r}, t)\right|_{\substack{r=R \\
z=-L}}+\left.\frac{\alpha_{1 \varphi}^{2}}{2} S_{\varphi \varphi 1010210}(\vec{r}, t)\right|_{z=-L}-\left.\frac{\alpha_{1 r}^{2}}{2} S_{r r 0010100}(\vec{r}, t)\right|_{z=-L}-\left.S_{r r 0000100}(\vec{r}, t)\right|_{r=R} \times \\
& \times \frac{V_{0}^{2}}{2}+\alpha_{1 z} \operatorname{tr} \varphi L-\left.\alpha_{1 r} r S_{t r 0010210}(\vec{r}, t)\right|_{\substack{r=R \\
z=-L}}+\left.\alpha_{1 r}^{2} \frac{r}{2} S_{r r 0010210}(\vec{r}, t)\right|_{\substack{r=R \\
z=-L}}+\left.S_{t r 0010210}(\vec{r}, t)\right|_{\substack{r=R \\
z=-L}} \times \\
& \times \alpha_{1 r} r+\alpha_{1 \varphi}^{2} \frac{r}{2} S_{\varphi \varphi 0000210}(\vec{r}, t)_{\substack{r=R \\
z=-L}}+\alpha_{1 r} \varphi L^{2} \frac{t}{2}+\left.\alpha_{1 r} S_{t r 1010210}(\vec{r}, t)\right|_{\substack{r=R \\
z=-L}}+\alpha_{1 r} \varphi t L\left(R+\frac{L}{2}\right)+ \\
& +\left.\alpha_{1 z}^{2} \frac{r}{2} S_{z z 0110210}(\vec{r}, t)\right|_{\substack{r=R \\
z=-L}}-\left.\alpha_{1 r} S_{t r 1010210}(\vec{r}, t)\right|_{\substack{r=R \\
z=-L}}+\left.\frac{\alpha_{1 r}^{2}}{2} S_{r r 0010100}(\vec{r}, t)\right|_{\substack{r=R \\
z=-L}}+\left.S_{r r 0010100}(\vec{r}, t)\right|_{\substack{r=R \\
z=-L}} \times \\
& \times \frac{\alpha_{1 r}^{2}}{2}-\alpha_{1 z} t \varphi R L-\left.\frac{\alpha_{1 r}^{2}}{2} S_{r r 1010210}(\vec{r}, t)\right|_{\substack{r=R \\
z=-L}}+\left.\frac{\alpha_{1 z}^{2}}{2} S_{z z 0000210}(\vec{r}, t)\right|_{r=R}+\int_{z=-L}^{t} \int_{0}^{\varphi} \int_{-L}^{0} \frac{P}{\rho} d w d v d \tau+ \\
& \left.\left.+\left.\frac{\alpha_{1 z}^{2}}{2} S_{r r 0000100}(\vec{r}, t)\right|_{\substack{r=R \\
z=-L}}+\left.\frac{\alpha_{1 \varphi}^{2}}{2} S_{\varphi \varphi 0010210}(\vec{r}, t)\right|_{\substack{r=R \\
z=-L}}\right]-\int_{0}^{t} \int_{0}^{r} \int_{0}^{\varphi} \int_{0}^{\varphi} \frac{P}{\rho} d w d v d u d \tau\right\}, \\
& v_{1 \varphi}=\alpha_{1 \varphi}+\frac{v}{2 L R}\left(\alpha_{1 r} \operatorname{tr} \varphi z-\alpha_{1 \varphi} t r^{2} \varphi^{2}+\alpha_{1 \varphi} S_{r r 2110100}(\vec{r}, t)-\alpha_{1 r}^{2} \frac{r^{2}}{2} S_{r r 0110210}(\vec{r}, t)+\right. \\
& +\alpha_{1 \varphi} S_{t t 2110210}(\vec{r}, t)-\alpha_{1 r}^{2} S_{r r 2110100}(\vec{r}, t)+\frac{\alpha_{1 r}^{2}}{2} S_{r r 2110210}(\vec{r}, t)+\frac{\alpha_{1 z}^{2}}{2} S_{r r 2110100}(\vec{r}, t)+\frac{\alpha_{1 \varphi}^{2}}{2} \times
\end{aligned}
$$


$\times S_{r r 1010100}(\vec{r}, t)+\frac{\alpha_{1 z}^{2}}{2} S_{z z 2110210}(\vec{r}, t)+\left[\left.\alpha_{1 r}^{2} \frac{r^{2}}{2} S_{r r 0110200}(\vec{r}, t)\right|_{\varphi=2 \pi}+\left.\alpha_{1 \varphi} S_{t r 2110210}(\vec{r}, t)\right|_{\varphi=2 \pi}-\right.$ $-\left.\alpha_{1 \varphi} S_{t r 2110210}(\vec{r}, t)\right|_{\varphi=2 \pi}+\left.\frac{\alpha_{1 r}^{2}}{2} S_{r r 2110100}(\vec{r}, t)\right|_{\varphi=2 \pi}-\pi \alpha_{1 r} t z^{2}-\left.\frac{\alpha_{1 z}^{2}}{2} S_{z z 2110210}(\vec{r}, t)\right|_{\varphi=2 \pi}+$ $\left.+2 \alpha_{1 \varphi} \pi^{2} t r^{2}-\left.\frac{\alpha_{1 \varphi}^{2}}{2} S_{r r 1010100}(\vec{r}, t)\right|_{\varphi=2 \pi}-\left.\frac{\alpha_{1 z}^{2}}{2} S_{r r 2100100}(\vec{r}, t)\right|_{\varphi=2 \pi}\right] \frac{\varphi}{2 \pi}-(2 \pi-\varphi) \omega^{2} r^{4} t \frac{\varphi}{4}+$ $+\frac{z}{L}\left\{\left.\alpha_{1 \varphi} S_{t \varphi 2110200}(\vec{r}, t)\right|_{z=L}+\left.\alpha_{1 \varphi} S_{t r 2110200}(\vec{r}, t)\right|_{z=L}-\left.\alpha_{1 \varphi} S_{t r 2110210}(\vec{r}, t)\right|_{z=L}+S_{r r 0110210}(\vec{r}, t) \times\right.$ $\times \alpha_{1 r}^{2} \frac{r^{2}}{2}-\left.\frac{\alpha_{1 r}^{2}}{2} S_{r r 2110210}(\vec{r}, t)\right|_{z=L}-\left.\alpha_{1 r}^{2} S_{r r 2110100}(\vec{r}, t)\right|_{z=L}-\left.\frac{\alpha_{1 z}^{2}}{2} S_{r r 2100100}(\vec{r}, t)\right|_{z=L}-\alpha_{1 r} r t \varphi \times$ $\times L-\left.\frac{\alpha_{1 z}^{2}}{2} S_{z z 2110211}(\vec{r}, t)\right|_{z=L}-\left.\frac{\alpha_{1 \varphi}^{2}}{2} S_{r r 1010100}(\vec{r}, t)\right|_{z=L}-\frac{\varphi}{2 \pi}\left[\left.\alpha_{1 r}^{2} S_{r r 2110100}(\vec{r}, t)\right|_{\substack{\varphi=2 \pi \\ z=L}}-2 \pi \alpha_{1 r} \times\right.$ $\times \operatorname{tr} L-\left.\alpha_{1 \varphi} S_{t r 2110210}(\vec{r}, t)\right|_{\substack{\varphi=2 \pi \\ z=L}}+\left.\alpha_{1 r}^{2} \frac{r^{2}}{2} S_{r r 0110100}(\vec{r}, t)\right|_{\substack{\varphi=2 \pi \\ z=L}}+\left.\alpha_{1 \varphi} S_{t r 2110100}(\vec{r}, t)\right|_{\substack{\varphi=2 \pi \\ z=L}}-\frac{\alpha_{1 r}^{2}}{2} \times$ $\times\left. S_{r r 2110210}(\vec{r}, t)\right|_{\substack{\varphi=2 \pi \\ z=L}}-\left.\frac{\alpha_{1 z}^{2}}{2} S_{z z 2100210}(\vec{r}, t)\right|_{\substack{\varphi=2 \pi \\ z=L}}-\left.\frac{\alpha_{1 \varphi}^{2}}{2} S_{r r 1010100}(\vec{r}, t)\right|_{\substack{\varphi=2 \pi \\ z=L}}-\left.S_{r r 2102100100}(\vec{r}, t)\right|_{\substack{\varphi=2 \pi \\ z=L}} \times$ $\left.\left.\left.\times \frac{\alpha_{1 z}^{2}}{2}\right]+(2 \pi-\varphi) \omega^{2} r^{4} t \frac{\varphi}{4}\right\}-\int_{0}^{t} \int_{0}^{r} u \int_{0}^{z} \frac{P}{\rho} d w d u d \tau\right)$ $v_{1 z}=\alpha_{1 z}+\frac{v}{2 R L}\left\{\alpha_{1 z} t \varphi \frac{z^{2}}{2}\left(\frac{\varphi}{2}+1\right)-\frac{\alpha_{1 r}^{2}}{2} S_{r r 1110210}(\vec{r}, t)+\alpha_{1 r} t r^{2} \frac{\varphi^{2}}{4}-S_{111110100}(\vec{r}, t) \times\right.$ $\times \frac{\alpha_{1 r}^{2}}{2}-\alpha_{1 z} S_{t r 1110210}(\vec{r}, t)-\frac{\alpha_{1 \varphi}^{2}}{2} S_{\varphi \varphi 0110210}(\vec{r}, t)+\frac{\alpha_{1 z}^{2}}{2} S_{z z 1110210}(\vec{r}, t)-\alpha_{1 z} S_{r r 1110100}(\vec{r}, t)-$ $-\frac{\alpha_{1 z}^{2}}{2} S_{r r 1110100}(\vec{r}, t)-\frac{\alpha_{1 \varphi}^{2}}{2} S_{r r 0110100}(\vec{r}, t)-\frac{z}{L}\left[\left.\alpha_{1 z} S_{t z 1110210}(\vec{r}, t)\right|_{z=-L}+\left.S_{r r 1110210}(\vec{r}, t)\right|_{z=-L} \times\right.$ $\times \frac{\alpha_{1 r}^{2}}{2}-\alpha_{1 z} t \varphi^{2} \frac{L^{2}}{4}-\left.\frac{\alpha_{1 \varphi}^{2}}{2} S_{r r 1010100}(\vec{r}, t)\right|_{z=-L}+\frac{\alpha_{1 r}^{2}}{2} S_{r r 0110210}(\vec{r}, t)-\left.\frac{\alpha_{1 z}^{2}}{2} S_{r r 1100100}(\vec{r}, t)\right|_{z=-L}-$ $\left.-\alpha_{1 z} L^{2} \frac{t}{2}-\left.\alpha_{1 z}^{2} \frac{L}{2} S_{z z 1100200}(\vec{r}, t)\right|_{z=-L}-\left.\frac{\alpha_{1 z}^{2}}{2} S_{z z 1110211}(\vec{r}, t)\right|_{z=-L}-\left.\frac{\alpha_{1 \varphi}^{2}}{2} S_{\varphi \varphi 1010210}(\vec{r}, t)\right|_{z=-L}\right]-$ $\left.-\int_{0}^{t} \int_{0}^{r}(r-u) \int_{0}^{\varphi}(\varphi-v) \frac{P}{\rho} d v d u d \tau\right\}$ 
Values of parameters ai, bi and ci could be determined by the following relations

$$
\begin{aligned}
& a_{1}=\frac{R^{2}}{4} \mu_{012 r 12}-\frac{1}{2} \mu_{112 r 01}-\frac{1}{8} \mu_{111 r 12}-\frac{R}{4} \mu_{112 r 12}+\frac{R}{4} \mu_{012 r 01}-\frac{L}{8} \widetilde{\mu}_{211 r 12}-\frac{L}{4} \widetilde{\mu}_{111 r 01}+ \\
& +L \frac{R^{2}}{8} \widetilde{\mu}_{011 r 12}+L \frac{R}{4} \widetilde{\mu}_{011 r 10}+L \frac{R}{4} \widetilde{\mu}_{011 r 01}-L \frac{R}{4} \widetilde{\mu}_{111 r 02 i j k \rho l m} \quad \mu_{i j k \rho l m}=\int_{0}^{\Theta}(\Theta-t) \int_{0}^{R}(R-r)^{i} \times \\
& \int_{0}^{2 \pi}(2 \pi-\varphi)^{j} \int_{-L}^{L}(L-z)^{k}\left(\frac{\partial v}{\partial \rho}\right)^{l} \frac{d z}{v^{m}} d \varphi d r d t \quad \widetilde{\mu}_{i j k \rho l m}=\int_{0}^{\Theta}(\Theta-t) \int_{0}^{R}(R-r)^{i} \int_{0}^{2 \pi}(2 \pi-\varphi)^{j} \times \\
& \times \int_{-L}^{0}(L+z)^{k}\left(\frac{\partial v}{\partial \rho}\right)^{l} \frac{d z}{v^{m}} d \varphi d r d t \quad a_{2}=\frac{R^{2}}{4} \mu_{010 \varphi 12}-\frac{1}{8} \mu_{212 \varphi 12}-\frac{1}{2} \mu_{001 \varphi 12}-\mu_{011 \varphi 12} \times \\
& \times L \frac{R}{4}-\frac{L}{4} \widetilde{\mu}_{111 \varphi 12}+L \frac{R^{2}}{8} \widetilde{\mu}_{010 \varphi 12}-L \frac{R}{4} \widetilde{\mu}_{011 \varphi 12} \quad a_{3}=\frac{R^{2}}{4} \mu_{012 z 12}-R \mu_{011 z 01}-\frac{1}{4} \mu_{210 z 01}- \\
& -\frac{1}{8} \mu_{212 z 12}+L \frac{R^{2}}{8} \tilde{\mu}_{011 z 12}+L \frac{R}{4} \widetilde{\mu}_{010 z 12}+L \frac{R}{4} \mu_{010 z 01} \quad a_{4}=\frac{R^{2}}{4} \mu_{012 z 01}-\frac{1}{4} \mu_{211 t 12}+\frac{R^{2}}{4} \times \\
& \times \mu_{012 t 12}-\frac{1}{4} \mu_{212 t 01}-8 R^{3} L \Theta^{2} \frac{\pi^{2}}{3}-\frac{R}{2} \mu_{112 t 12}-\frac{R}{2} \mu_{112 t 01}-3 \Theta^{2} R^{2} L^{2} \frac{\pi^{2}}{2}-\frac{L}{4} \tilde{\mu}_{211 t 12}-\Theta^{2} \times \\
& \times R L^{3} \frac{\pi^{2}}{2}-\frac{L}{4} \widetilde{\mu}_{211 t 01}+L \frac{R^{2}}{4} \widetilde{\mu}_{011 t 12}-L \frac{R^{2}}{2} \widetilde{\mu}_{011 t 01}+R \widetilde{\mu}_{101 t 01}-R \frac{L}{2} \widetilde{\mu}_{101 t 12} \quad a_{5}=V_{0}^{2} R \frac{L}{4} \times \\
& \times \mu_{011 t 01}-V_{0}^{2} R \frac{L^{2}}{4} \hat{\mu}_{010 t 01}+V_{0}^{2} R \frac{L^{2}}{4} \mu_{010 t 01}+\frac{L}{2} \int_{0}^{\Theta}(\Theta-t) \int_{0}^{2 \pi}(2 \pi-\varphi) \int_{-L}^{0} \frac{P}{\rho} d z d \varphi d t-\int_{0}^{\Theta}(\Theta-t) \times \\
& \times \int_{0}^{R}(R-r) \int_{0}^{2 \pi}(2 \pi-\varphi) \int_{-L}^{L}(L-z) \frac{P}{\rho} d z d \varphi d t+\int_{0}^{\Theta} \int_{0}^{R}(R-r) \int_{0}^{2 \pi}(2 \pi-\varphi) \int_{-L}^{L}(L-z) \frac{P}{\rho} d z d \varphi d r \times \\
& \times(\Theta-t) d t ; \quad \hat{\mu}_{i j k \rho l m}=\int_{0}^{\Theta}(\Theta-t) \int_{0}^{R}(R-r)^{i} \int_{0}^{2 \pi}(2 \pi-\varphi)^{j} \int_{0}^{L}(L-z)^{k}\left(\frac{\partial v}{\partial \rho}\right)^{l} \frac{d z}{v^{m}} d \varphi d r d t \\
& b_{1}=\frac{1}{4} \mu_{222 r 01}+\frac{1}{8} \mu_{322 r 12}-\frac{1}{4} \mu_{322 r 01}+\pi^{2} \mu_{312 r 01}-\frac{\pi^{2}}{2} \mu_{312 r 12}-\pi^{2} \mu_{012 r 01} \frac{R^{3}}{6}+\hat{\mu}_{211 r 01} \times \\
& \times L \frac{\pi}{4}-\frac{L}{4} \hat{\mu}_{211 r 01}-\frac{L}{8} \hat{\mu}_{321 r 12}-\frac{L}{4}\left(\pi+\frac{1}{2}\right) \hat{\mu}_{321 r 01}+L \frac{\pi}{4} \hat{\mu}_{311 r 12} \quad b_{2}=\frac{1}{2} \mu_{211 r 01}-\frac{1}{2} \hat{\mu}_{211 r 01}+ \\
& +L \frac{\pi}{4} \hat{\mu}_{201 r 01}-\frac{\pi^{2}}{2} \mu_{202 r 01} \quad b_{3}=\frac{1}{4} \mu_{321 z 01}+\frac{1}{8} \mu_{322 z 12}-\pi^{2} \mu_{311 z 01}-\frac{\pi^{2}}{4} \mu_{312 z 12}-\frac{L}{4} \mu_{320 z 01}-
\end{aligned}
$$




$$
\begin{gathered}
-\frac{L}{8} \mu_{321 z 12}+L \frac{\pi}{4} \hat{\mu}_{310 z 01}+L \frac{\pi}{4} \hat{\mu}_{310 z 12} \quad b_{4}=-\pi^{2} \Theta^{2} R^{2} \frac{L^{2}}{4}-2 \pi^{2} \Theta^{2} R L^{3} \quad b_{5}=\frac{1}{2} \mu_{322 t 12}+ \\
+\frac{1}{2} \mu_{322 t 01}-\pi \mu_{312 t 01}-\pi \mu_{322 t 12}-2 \pi^{3} \Theta^{2} R^{3} L-\frac{L}{4} \hat{\mu}_{321 t 01}-\frac{L}{4} \hat{\mu}_{321 t 01}-\frac{L}{4} \hat{\mu}_{321 t 12}+L \frac{\pi}{2} \hat{\mu}_{311 t 12}+ \\
+L \frac{\pi}{2} \hat{\mu}_{311 t 01} b_{6}=\omega^{2} R^{5} \Theta^{2} L \frac{\pi^{3}}{10}-\int_{0}^{\Theta}(\Theta-t) \int_{0}^{R} r(R-r) \int_{0}^{2 \pi} \int_{-L}^{L}(L-z) \frac{P}{\rho} d z d \varphi d r d t \\
c_{1}=-\frac{1}{8} \mu_{211 r 12}-\frac{1}{8} \mu_{211 r 01}-\frac{L}{8} \mu_{021 r 01}-\frac{L}{16} \tilde{\mu}_{221 r 12} c_{2}=-\frac{1}{8} \mu_{122 \varphi 12}-\frac{1}{8} \mu_{122 \varphi 01}- \\
-\frac{L}{8} \tilde{\mu}_{211 \varphi 01}-\frac{L}{8} \tilde{\mu}_{211 \varphi 12} c_{3}=\frac{1}{8} \mu_{222 z 12}-\frac{1}{8} \mu_{222 z 01}-\frac{L^{2}}{16} \mu_{220 z 01}-\frac{1}{16} \tilde{\mu}_{220 z 12}-\frac{L^{2}}{16} \tilde{\mu}_{220 z 12} \\
c_{4}=\Theta^{2} R^{3} \frac{\pi^{3}}{18} ; \quad c_{5}=\frac{L}{4} \tilde{\mu}_{221 t 12}-\frac{1}{8} \mu_{222 t 12}+\Theta^{2} R L^{3} \frac{\pi^{3}}{6}-\frac{1}{8} \mu_{221 t 01}-\frac{L}{8} \tilde{\mu}_{221 t 01}-L^{3} \Theta^{2} R \frac{\pi}{12} \\
c_{6}=-\int_{0}^{\Theta}(\Theta-t) \int_{0}^{R}(R-r)^{2} \int_{0}^{2 \pi}(2 \pi-\varphi) \int_{-L}^{L} \frac{P}{4 \rho} d z d \varphi d r d t
\end{gathered}
$$

Second-order approximations of components of velocity of flow of composition of gases (gas-carrier and materials in gas phase, which are used for formation epitaxial layer) $\mathrm{vr}, \mathrm{v} \varphi$ and $\mathrm{vz}$ could be written as

$$
\begin{aligned}
& v_{2 r}=\alpha_{2 r}+v_{1 r}+\left\{\iint_{0}^{t} \int_{0}^{r} \int_{0}^{\varphi}(\varphi-v) \int_{0}^{z}\left(\alpha_{2 r}+v_{1 r}\right) d w d v d u d \tau+\int_{0}^{t} \int_{0}^{r} \int_{0}^{\varphi} \int_{0}^{z}\left(\alpha_{2 r}+v_{1 r}\right) d w d v d u d \tau+\right. \\
& +\int_{0}^{t} \int_{0}^{r} \int_{0}^{\varphi} \int_{0}^{z}\left(\alpha_{2 z}+v_{1 z}\right) d w d v d u d \tau-\int_{0}^{t} \int_{0}^{r}(r-u) \int_{0}^{\varphi}\left(\alpha_{2 r}+v_{1 r}\right) d v d u d \tau-\int_{0}^{r}(r-u) \int_{0}^{\varphi z} \int_{0}^{z}\left(\alpha_{2 r}+v_{1 r}\right) \times \\
& \times(z-w) \frac{d w}{v} d v d u-\int_{0}^{t} \int_{0}^{r}(r-u) \int_{0}^{\varphi} \int_{0}^{z}(z-w)\left(\alpha_{2 r}+v_{1 r}\right) \frac{\partial v}{\partial \tau} d w d v d u d \tau-\frac{1}{2} \iint_{0}^{t} \int_{0}^{r} \int_{0}^{\varphi z} \int_{0}^{z}(z-w) \times \\
& \times\left(\alpha_{2 r}+v_{1 r}\right)^{2} \frac{d w}{v} d v d u d \tau-\frac{1}{2} \int_{0}^{t} \int_{0}^{r}(r-u) \int_{0}^{\varphi} \int_{0}^{z}\left(\alpha_{2 z}+v_{1 z}\right) \frac{d w}{v} d v d u d \tau-\frac{1}{2} \int_{0}^{t} \int_{0}^{r}(r-u) \int_{0}^{\varphi z} \int_{0}^{z}(z- \\
& -w)\left(\alpha_{2 r}+v_{1 r}\right)^{2} \frac{\partial v}{\partial u} \frac{d w}{v^{2}} d v d u d \tau-\frac{1}{2} \int_{0}^{t} \int_{0}^{r} \frac{r-u}{u} \int_{0}^{\varphi} \int_{0}^{z}(z-w)\left(\alpha_{2 \varphi}+v_{1 \varphi}\right)^{2} \frac{\partial v}{\partial v} \frac{d w}{v^{2}} d v d u d \tau+ \\
& +r \int_{0}^{t} \int_{0}^{R} \int_{0}^{\varphi} \int_{0}^{z}(z-w)\left(\alpha_{2 r}+v_{1 r}\right) \frac{\partial v}{\partial \tau} \frac{d w}{v^{2}} d v d u d \tau+\int_{0}^{R} r \int_{0}^{\varphi} \int_{0}^{z}(z-w)\left(\alpha_{2 r}+v_{1 r}\right) \frac{d w}{v} d v d r- \\
& -\int_{0}^{t} \int_{0}^{R} \int_{0}^{\varphi} \int_{0}^{z}\left(\alpha_{2 r}+v_{1 r}\right) d w d v d u d \tau-\frac{1}{2} \int_{0}^{t} \int_{0}^{r}(r-u) \int_{0}^{\varphi} \int_{0}^{z}(z-w)\left(\alpha_{2 z}+v_{1 z}\right)^{2} \frac{\partial v}{\partial w} \frac{d w}{v^{2}} d v d u d \tau+ \\
& +\frac{r}{2} \int_{0}^{t} \int_{0}^{R} \int_{0}^{\varphi} \int_{0}^{z}(z-w)\left(\alpha_{2 r}+v_{1 r}\right)^{2} \frac{\partial v}{\partial u} \frac{d w}{v^{2}} d v d u d \tau+\int_{0}^{t} \int_{0}^{R} r \int_{0}^{\varphi}\left(\alpha_{2 r}+v_{1 r}\right) d v d r d \tau-\int_{0}^{t} \int_{0}^{R}(R-u) \times
\end{aligned}
$$


$\times \int_{0}^{\varphi}\left(\alpha_{2 r}+v_{1 r}\right) d v d u d \tau-\iint_{0}^{t} \int_{0}^{R \varphi} \int_{0}^{\varphi} \int_{0}^{z}\left(\alpha_{2 z}+v_{1 z}\right) d w d v d u d \tau-\int_{0}^{R \varphi} \int_{0}^{p} \int_{0}^{z}(z-w)\left(\alpha_{2 r}+v_{1 r}\right) \frac{d w}{v} d v \times$ $\times u d u+\frac{r}{2} \iint_{0}^{t} \int_{0}^{t \varphi} \int_{0} \int_{0}^{z}(z-w)\left(\alpha_{2 z}+v_{1 z}\right)^{2} \frac{\partial v}{\partial w} \frac{d w}{v^{2}} d v d u d \tau-\frac{1}{2} \iint_{0}^{t} \int_{0}^{R} u \int_{0}^{\varphi} \int_{0}^{\varphi z}(z-w)\left(\alpha_{2 r}+v_{1 r}\right)^{2} \frac{\partial v}{\partial u} \times$ $\times \frac{d w}{v^{2}} d v d u d \tau-\int_{0}^{t} \int_{0}^{R} u \int_{0}^{\varphi} \int_{0}^{z}(z-w)\left(\alpha_{2 r}+v_{1 r}\right) \frac{\partial v}{\partial \tau} \frac{d w}{v^{2}} d v d u d \tau+\frac{r}{2} \iint_{0}^{t} \int_{0}^{R} \frac{1}{u} \int_{0}^{\varphi z} \int_{0}^{z}\left(\alpha_{2 \varphi}+v_{1 \varphi}\right)^{2} \frac{\partial v}{\partial v} \times$ $\times \frac{d w}{v^{2}} d v d u d \tau-\iint_{0}^{t} \int_{0}^{R} \int_{0}^{\varphi z}\left(\alpha_{2 z}+v_{1 z}\right)^{2} \frac{\partial v}{\partial w} \frac{d w}{2 v^{2}} d v d u d \tau-\iint_{0}^{t} \int_{0}^{R} \int_{0} \int_{0}^{z}\left(\alpha_{2 z}+v_{1 z}\right)^{2} \frac{d w}{2 v} d v d u d \tau-$ $-\frac{1}{2} \iint_{0}^{t} \int_{0}^{R \varphi} \int_{0} \int_{0}^{z}(z-w)\left(\alpha_{2 \varphi}+v_{1 \varphi}\right)^{2} \frac{\partial v}{\partial v} \frac{d w}{v^{2}} d v d u d \tau+\int_{0}^{t} \int_{0}^{R \varphi} \int_{0} \int_{0}^{z}(z-w)\left(\alpha_{2 r}+v_{1 r}\right)^{2} \frac{d w}{2 v} d v d u d \tau-$ $-R \int_{0}^{t} \int_{0}^{R \varphi} \int_{0}^{\varphi}\left(\alpha_{2 r}+v_{1 r}\right) d v d u d \tau-\frac{z}{L}\left[\int_{0}^{r}(r-u) \int_{0}^{\varphi} \int_{-L}^{0}(L+w)\left(\alpha_{2 r}+v_{1 r}\right) \frac{d w}{v} d v d u+\int_{0}^{t r} \int_{0}^{r}(r-u) \times\right.$ $\times \int_{0}^{\varphi} \int_{-L}^{0}(L+w)\left(\alpha_{2 r}+v_{1 r}\right) \frac{\partial v}{\partial \tau} \frac{d w}{v^{2}} d v d u d \tau+\int_{0}^{t} \int_{0}^{t} \int_{0}^{\varphi} \int_{-L}^{0}\left(\alpha_{2 r}+v_{1 r}\right) d w d v d u d \tau+\frac{1}{2} \int_{0}^{t} \int_{0}^{r}(r-u) \times$ $\times \int_{0}^{\varphi} \int_{-L}^{0}(L+w)\left(\alpha_{2 r}+v_{1 r}\right)^{2} \frac{\partial v}{\partial u} \frac{d w}{v^{2}} d v d u d \tau+\frac{1}{2} \int_{0}^{t} \int_{0}^{r} \int_{0}^{\varphi} \int_{-L}^{0}(L+w)\left(\alpha_{2 \varphi}+v_{1 \varphi}\right)^{2} \frac{\partial v}{\partial v} \frac{d w}{v^{2}} d v \times$ $\times \frac{r-u}{u} d u d \tau+\int_{0}^{t} \int_{0}^{r \varphi} \int_{0}^{0} \int_{-L}^{0}\left(\alpha_{2 z}+v_{1 z}\right) d w d v d u d \tau+\int_{0}^{t} \int_{0}^{r} \int_{0}^{\varphi} \int_{-L}^{0}(L+w)\left(\alpha_{2 r}+v_{1 r}\right) \frac{d w}{2 v} d v d u d \tau+$ $+\frac{1}{2} \int_{0}^{t} \int_{0}^{R \varphi} \int_{0}^{\infty} \int_{-L}^{0}(z-w)\left(\alpha_{2 \varphi}+v_{1 \varphi}\right)^{2} \frac{\partial v}{\partial v} \frac{d w}{v^{2}} d v d u d \tau-\frac{V_{0}^{2}}{2} \iint_{0}^{t} \int_{0}^{R \varphi} \int_{0} \int_{0} \frac{d w}{v} d v d u d \tau-r \iint_{0}^{t} \int_{0}^{R \varphi} \int_{0}^{0} \int_{-L}^{0}(L+w) \times$ $\times\left(\alpha_{2 r}+v_{1 r}\right) \frac{\partial v}{\partial \tau} \frac{d w}{v^{2}} d v d u d \tau-\frac{r}{2} \iint_{0}^{t} \int_{0}^{R} \frac{1}{u} \int_{0}^{\varphi} \int_{-L}^{0}\left(\alpha_{2 \varphi}+v_{1 \varphi}\right)^{2} \frac{\partial v}{\partial v} \frac{d w}{v^{2}} d v d u d \tau-\frac{r}{2} \iint_{0}^{t} \int_{0}^{R \varphi} \int_{0}^{0} \int_{-L}^{0}(L+w) \times$ $\times\left(\alpha_{2 r}+v_{1 r}\right)^{2} \frac{\partial v}{\partial u} \frac{d w}{v^{2}} d v d u d \tau-\int_{0}^{t} \int_{0}^{\varphi} \int_{-L}^{0}(L+w)\left(\alpha_{2 r}+v_{1 r}\right) d w d v d \tau-r \iint_{0}^{R} \int_{0}^{\varphi} \int_{-L}^{0}\left(\alpha_{2 r}+v_{1 r}\right)(L+$ $+w) \frac{d w}{v} d v d u-\frac{1}{2} \iiint_{0}^{t} \int_{0}^{R} \int_{-L}^{0}(L+w)\left(\alpha_{2 r}+v_{1 r}\right)^{2} \frac{d w}{v} d v d u d \tau-\frac{r}{2} \iiint_{0}^{t} \int_{0}^{t} \int_{-L}^{0}(L+w)\left(\alpha_{2 z}+v_{1 z}\right)^{2} \times$ $\times \frac{\partial v}{\partial w} \frac{d w}{v^{2}} d v d u d \tau-\iint_{0}^{t} \int_{0}^{\rho} \int_{0}^{0}\left(\alpha_{-L}^{0}+v_{1 r}\right) d w d v d u d \tau-\frac{1}{2} \iint_{0}^{t} \int_{0}^{R} \int_{0}^{\varphi} \int_{-L}^{0}\left(\alpha_{2 z}+v_{1 z}\right)^{2} \frac{d w}{v} d v d u d \tau+$ $+\int_{0}^{t} \int_{0}^{R} u \int_{0}^{\varphi} \int_{-L}^{0}(L+w)\left(\alpha_{2 r}+v_{1 r}\right) \frac{\partial v}{\partial \tau} \frac{d w}{v^{2}} d v d u d \tau-\frac{1}{2} \iint_{0}^{t} \int_{0}^{R \varphi} \int_{0}^{\varphi} \int_{-L}^{0}\left(\alpha_{2 z}+v_{1 z}\right)^{2} \frac{\partial v}{\partial w} \frac{d w}{v^{2}} d v d u d \tau+$ 
$+\int_{0}^{R} u \int_{0}^{\varphi} \int_{-L}^{0}(L+w) \frac{v_{r}}{v} d w d v d u-\int_{0}^{t} \int_{0}^{R} \int_{0}^{\varphi} \int_{-L}^{0}\left(\alpha_{2 z}+v_{1 z}\right) d w d v d u d \tau+\frac{1}{2} \int_{0}^{t} \int_{0}^{R} \int_{0}^{\varphi} \int_{0}^{0}\left(\alpha_{2 \varphi}+v_{1 \varphi}\right)^{2} \frac{\partial v}{\partial v}(L+$ $\left.+w) \frac{d w}{v^{2}} d v d u d \tau-\int_{0}^{t} \int_{0}^{r} \int_{0}^{\varphi} \int_{0}^{z} \frac{P}{\rho} d w d v d \tau\right\} \frac{v}{2 R L}$

$v_{2 \varphi}=\alpha_{2 \varphi}+v_{1 \varphi}+\frac{v}{2 L R}\left(\int_{0}^{t} \int_{0}^{r} \int_{0}^{\varphi} \int_{0}^{z}(z-w)\left(\alpha_{2 r}+v_{1 r}\right) d w d v d u d \tau-r \int_{0}^{t} \int_{0}^{\varphi z} \int_{0}^{z}\left(\alpha_{2 r}+v_{1 r}\right)(z-\right.$ $-w) d w d v d \tau+\int_{0}^{t} \int_{0}^{r} \int_{0}^{\varphi} \int_{0}^{z}\left(\alpha_{2 r}+v_{1 r}\right) d w d v d u d \tau-\int_{0}^{t} \int_{0}^{r} u \int_{0}^{\varphi}(\varphi-v)\left(\alpha_{2 \varphi}+v_{1 \varphi}\right) d v d u d \tau+$ $+\int_{0}^{r} u^{2} \int_{0}^{\varphi}(\varphi-v) \int_{0}^{z}(z-w)\left(\alpha_{2 \varphi}+v_{1 \varphi}\right) \frac{d w}{v} d v d u+\frac{r^{2}}{2} \int_{0}^{t} \int_{0}^{\varphi}(\varphi-v) \int_{0}^{z}(z-w)\left(\alpha_{2 r}+v_{1 r}\right)^{2} \times$ $\times \frac{d w}{v} d v d \tau+\int_{0}^{t} \int_{0}^{r} u^{2} \int_{0}^{\varphi}(\varphi-v) \int_{0}^{z}(z-w)\left(\alpha_{2 \varphi}+v_{1 \varphi}\right) \frac{\partial v}{\partial \tau} \frac{d w}{v^{2}} d v d u d \tau-\int_{0}^{t} \int_{0}^{r} u^{2} \int_{0}^{\varphi}(\varphi-v) \times$ $\times \int_{0}^{z}(z-w)\left(\alpha_{2 r}+v_{1 r}\right)^{2} \frac{d v}{v} d u d \tau+\frac{1}{2} \int_{0}^{t} \int_{0}^{r} \int_{0}^{\varphi}(\varphi-v) \int_{0}^{z}(z-w)\left(\alpha_{2 r}+v_{1 r}\right)^{2} \frac{\partial v}{\partial u} \frac{d w}{v^{2}} d v \times$ $\times u^{2} d u d \tau+\frac{1}{2} \int_{0}^{t} \int_{0}^{r} u^{2} \int_{0}^{\varphi}(\varphi-v) \int_{0}^{z}\left(\alpha_{2 z}+v_{1 z}\right)^{2} \frac{d w}{v} d w d v d u d \tau+\frac{1}{2} \int_{0}^{t} \int_{0}^{r} \int_{0}^{\varphi} \int_{0}^{z}\left(\alpha_{2 \varphi}+v_{1 \varphi}\right)^{2} \times$ $\times(z-w) \frac{d w}{v} d v u d u d \tau+\frac{1}{2} \int_{0}^{t} \int_{0}^{r} u^{2} \int_{0}^{\varphi}(\varphi-v) \int_{0}^{z}(z-w)\left(\alpha_{2 z}+v_{1 z}\right)^{2} \frac{\partial v}{\partial w} \frac{d w}{v^{2}} d v d u d \tau+$ $+\frac{\varphi}{2 \pi}\left[r \int_{0}^{t} \int_{0}^{2 \pi} \int_{0}^{z}(z-w)\left(\alpha_{2 r}+v_{1 r}\right) d w d v d \tau-\int_{0}^{t} \int_{0}^{r} \int_{0}^{2 \pi} \int_{0}^{z}\left(\alpha_{2 r}+v_{1 r}\right) d w d v d u d \tau-\int_{0}^{t} \int_{0}^{2 \pi} \int_{0}^{z}(z-\right.$ $-w)\left(\alpha_{2 r}+v_{1 r}\right) d w d u d \tau-\frac{r^{2}}{2} \int_{0}^{t} \int_{0}^{2 \pi}(2 \pi-v) \int_{0}^{z}(z-w)\left(\alpha_{2 r}+v_{1 r}\right)^{2} \frac{d w}{v} d v d \tau-\int_{0}^{r} u^{2} \times$ $\times \int_{0}^{2 \pi}(2 \pi-v) \int_{0}^{z}(z-w)\left(\alpha_{2 \varphi}+v_{1 \varphi}\right) \frac{d w}{v} d v d u-\int_{0}^{t} \int_{0}^{r} u^{2} \int_{0}^{2 \pi}(2 \pi-v) \int_{0}^{z}(z-w)\left(\alpha_{2 \varphi}+v_{1 \varphi}\right) \frac{d w}{v^{2}} \times$ $\times \frac{\partial v}{\partial \tau} d v d u d \tau+\int_{0}^{t} \int_{0}^{r} u \int_{0}^{2 \pi}(2 \pi-v)\left(\alpha_{2 \varphi}+v_{1 \varphi}\right) d v d u d \tau+\int_{0}^{t} \int_{0}^{r} u^{2} \int_{0}^{2 \pi}(2 \pi-v) \int_{0}^{z}\left(\alpha_{2 r}+v_{1 r}\right)^{2}(z-$ $-w) \frac{d w}{v} d w d v d u d \tau-\frac{1}{2} \int_{0}^{t} \int_{0}^{r} u^{2} \int_{0}^{2 \pi}(2 \pi-v) \int_{0}^{z}(z-w)\left(\alpha_{2 r}+v_{1 r}\right)^{2} \frac{\partial v}{\partial u} \frac{d w}{v^{2}} d v d u d \tau-$ $-\frac{1}{2} \int_{0}^{t} \int_{0}^{r} u^{2} \int_{0}^{2 \pi}(2 \pi-v) \int_{0}^{z}\left(\alpha_{2 z}+v_{1 z}\right)^{2} \frac{d w}{v} d v d u d \tau-\frac{1}{2} \int_{0}^{t} \int_{0}^{r} u^{2} \int_{0}^{2 \pi}(2 \pi-v) \int_{0}^{z}(z-w)\left(\alpha_{2 z}+v_{1 z}\right)^{2} \times$ $\left.\times \frac{\partial v}{\partial w} \frac{d w}{v^{2}} d v d u d \tau-\frac{1}{2} \int_{0}^{t} \int_{0}^{r} u \int_{0}^{2 \pi} \int_{0}^{z}(z-w)\left(\alpha_{2 \varphi}+v_{1 \varphi}\right)^{2} \frac{d w}{v} d v d u d \tau\right]-(2 \pi-\varphi) \omega^{2} r^{4} t \frac{\varphi}{4}+$ 
$+\frac{z}{L}\left\{r \int_{0}^{t} \int_{0}^{\varphi} \int_{0}^{L}(L-w)\left(\alpha_{2 r}+v_{1 r}\right) d w d v d \tau-\int_{0}^{t} \int_{0}^{r} \int_{0}^{\varphi} \int_{0}^{L}(L-w)\left(\alpha_{2 r}+v_{1 r}\right) d w d v d u d \tau-\frac{r^{2}}{2} \int_{0}^{t} \int_{0}^{\varphi}(\varphi-\right.$ $-v) \int_{0}^{L}(L-w)\left(\alpha_{2 r}+v_{1 r}\right)^{2} \frac{d w}{v} d v d \tau-\int_{0}^{r} u^{2} \int_{0}^{\varphi}(\varphi-v) \int_{0}^{L}(L-w)\left(\alpha_{2 \varphi}+v_{1 \varphi}\right) \frac{d w}{v} d v d u-\int_{0}^{r} u^{2} \times$ $\times \int_{0}^{\varphi}(\varphi-v) \int_{0}^{L}(L-w)\left(\alpha_{2 \varphi}+v_{1 \varphi}\right) \frac{d w}{v} d v d u-\int_{0}^{t} \int_{0}^{r} \int_{0}^{\varphi}(\varphi-v) \int_{0}^{L}(L-w)\left(\alpha_{2 \varphi}+v_{1 \varphi}\right) \frac{\partial v}{\partial \tau} \frac{d w}{v^{2}} d v \times$ $\times u^{2} d u d \tau-\frac{1}{2} \int_{0}^{t} \int_{0}^{r} u^{2} \int_{0}^{\varphi}(\varphi-v) \int_{0}^{L}(L-w)\left(\alpha_{2 r}+v_{1 r}\right)^{2} \frac{\partial v}{\partial u} \frac{d w}{v^{2}} d v d u d \tau-\int_{0}^{t} \int_{0}^{r} u^{2} \int_{0}^{\varphi}(\varphi-v) \int_{0}^{L}(L-$ $-w)\left(\alpha_{2 r}+v_{1 r}\right)^{2} \frac{d w}{v} d v d u d \tau-\int_{0}^{t} \int_{0}^{r} \int_{0}^{\varphi} \int_{0}^{L}\left(\alpha_{2 r}+v_{1 r}\right) d w d v d u d \tau-\frac{1}{2} \int_{0}^{t} \int_{0}^{r} \int_{0}^{\varphi}(\varphi-v) \int_{0}^{L}\left(\alpha_{2 z}+\right.$ $\left.+v_{1 z}\right)^{2} \frac{d w}{v} d v u^{2} d u d \tau-\frac{1}{2} \int_{0}^{t} \int_{0}^{r} u^{2} \int_{0}^{\varphi}(\varphi-v) \int_{0}^{L}(L-w)\left(\alpha_{2 z}+v_{1 z}\right)^{2} \frac{\partial v}{\partial w} \frac{d w}{v^{2}} d v d u d \tau-$ $-\frac{1}{2} \int_{0}^{t} \int_{0}^{r} u \int_{0}^{\varphi} \int_{0}^{L}(L-w)\left(\alpha_{2 \varphi}+v_{1 \varphi}\right)^{2} \frac{d w}{v} d v d u d \tau-\frac{\varphi}{2 \pi}\left[r \int_{0}^{t} \int_{0}^{2 \pi} \int_{0}^{L}(L-w)\left(\alpha_{2 r}+v_{1 r}\right)^{2} d w d v d \tau-\right.$ $-\frac{r^{2}}{2} \int_{0}^{t} \int_{0}^{2 \pi}(2 \pi-v) \int_{0}^{L}(L-w)\left(\alpha_{2 r}+v_{1 r}\right)^{2} \frac{d w}{v} d v d \tau-\int_{0}^{t} \int_{0}^{r} \int_{0}^{2 \pi} \int_{0}^{L}(L-w)\left(\alpha_{2 r}+v_{1 r}\right) d w d v d u d \tau-$ $-\int_{0}^{t} \int_{0}^{r} \int_{0}^{2 \pi} \int_{0}^{L}\left(\alpha_{2 r}+v_{1 r}\right) d w d v d u d \tau-\int_{0}^{t} \int_{0}^{r} u^{2} \int_{0}^{2 \pi}(2 \pi-v) \int_{0}^{L}(L-w)\left(\alpha_{2 \varphi}+v_{1 \varphi}\right) \frac{\partial v}{\partial \tau} \frac{d w}{v^{2}} d v d u d \tau-$ $-\int_{0}^{r} u^{2} \int_{0}^{2 \pi}(2 \pi-v) \int_{0}^{L}(L-w)\left(\alpha_{2 \varphi}+v_{1 \varphi}\right) \frac{d w}{v} d v d u-\frac{1}{2} \int_{0}^{t} \int_{0}^{r} u^{2} \int_{0}^{2 \pi}(2 \pi-v) \int_{0}^{L}(L-w)\left(\alpha_{2 r}+v_{1 r}\right)^{2} \times$ $\times \frac{\partial v}{\partial u} \frac{d w}{v^{2}} d v d u d \tau+\int_{0}^{t} \int_{0}^{r} u^{2} \int_{0}^{2 \pi}(2 \pi-v) \int_{0}^{L}(L-w)\left(\alpha_{2 r}+v_{1 r}\right)^{2} \frac{d w}{v} d v d u d \tau-\frac{1}{2} \int_{0}^{t} \int_{0}^{r} u^{2} \int_{0}^{2 \pi}(2 \pi-$ $-v) \int_{0}^{L}\left(\alpha_{2 z}+v_{1 z}\right)^{2} \frac{d w}{v} d v d u d \tau-\frac{1}{2} \int_{0}^{t} \int_{0}^{r} u^{2} \int_{0}^{2 \pi}(2 \pi-v) \int_{0}^{L}\left(\alpha_{2 z}+v_{1 z}\right)^{2} \frac{\partial v}{\partial w} \frac{d w}{v^{2}} d v d u d \tau-$ $\left.\left.\left.-\frac{1}{2} \int_{0}^{t} \int_{0}^{r} u \int_{0}^{2 \pi L} \int_{0}^{L}(L-w)\left(\alpha_{2 \varphi}+v_{1 \varphi}\right)^{2} \frac{d w}{v} d v d u d \tau\right]+\omega^{2} t r^{4}(2 \pi-\varphi) \frac{\varphi}{4}\right\}-\int_{0}^{t} \int_{0}^{r} \int_{0}^{z} \frac{P}{\rho} d w d u d \tau\right)$ $v_{2 z}=\alpha_{2 z}+v_{1 z}+\frac{v}{2 R L}\left\{\int_{0}^{t} \int_{0}^{r}(r-u) \int_{0}^{\varphi}(\varphi-v)\left(\alpha_{2 r}+v_{1 r}\right) d v d u d \tau+\int_{0}^{t} \int_{0}^{\varphi}(\varphi-v) \int_{0}^{z}\left(\alpha_{2 z}+v_{1 z}\right)(z-\right.$ $-w) d w d v d \tau+\int_{0}^{t} \int_{0}^{r} \frac{r-u}{u^{2}} \int_{0}^{\varphi z} \int_{0}^{z}(z-w)\left(\alpha_{2 z}+v_{1 z}\right) d w d v d u d \tau-\frac{1}{2} \int_{0}^{t} \int_{0}^{r}(r-u) \int_{0}^{\varphi}(\varphi-v) \int_{0}^{z}(z-$ 


$$
\begin{aligned}
& -w)\left(\alpha_{2 r}+v_{1 r}\right)^{2} \frac{\partial v}{\partial u} \frac{d w}{v^{2}} d v d u d \tau-\frac{1}{2} \int_{0}^{t} \int_{0}^{r} \int_{0}^{\varphi}(\varphi-v) \int_{0}^{z}(z-w)\left(\alpha_{2 \varphi}+v_{1 \varphi}\right)^{2} \frac{\partial v}{\partial v} \frac{d w}{v^{2}} d v \times \\
& \times \frac{1}{u} d u d \tau-\frac{1}{2} \int_{0}^{t} \int_{0}^{r}(r-u) \int_{0}^{\varphi}(\varphi-v) \int_{0}^{z}(z-w)\left(\alpha_{2 r}+v_{1 r}\right)^{2} \frac{d w}{v} d v d u d \tau-\int_{0}^{t} \int_{0}^{r}(r-u) \int_{0}^{\varphi}(\varphi-v) \times \\
& \times \int_{0}^{z}(z-w)\left(\alpha_{2 z}+v_{1 z}\right) \frac{\partial v}{\partial \tau} \frac{d w}{v^{2}} d v d u d \tau-\frac{1}{2} \int_{0}^{t} \int_{0}^{r}(r-u) \int_{0}^{\varphi}(\varphi-v) \int_{0}^{z}(z-w)\left(\alpha_{2 z}+v_{1 z}\right)^{2} \frac{\partial v}{\partial w} \times \\
& \times \frac{d w}{v^{2}} d v d u d \tau-\int_{0}^{r}(r-u) \int_{0}^{\varphi}(\varphi-v) \int_{0}^{z}(z-w)\left(\alpha_{2 z}+v_{1 z}\right) \frac{d w}{v} d v d u-\frac{1}{2} \int_{0}^{t} \int_{0}^{r}(r-u) \int_{0}^{\varphi}(\varphi-v) \times \\
& \times \int_{0}^{z}(z-w)\left(\alpha_{2 z}+v_{1 z}\right)^{2} \frac{d w}{v} d v d u d \tau-\frac{1}{2} \int_{0}^{t} \int_{0}^{r} \int_{0}^{\varphi}(\varphi-v) \int_{0}^{z}(z-w)\left(\alpha_{2 \varphi}+v_{1 \varphi}\right)^{2} \frac{d w}{v} d v \times \\
& \times \frac{1}{u} d u d \tau-\frac{z}{L}\left[\int_{0}^{r}(r-u) \int_{0}^{\varphi}(\varphi-v) \int_{-L}^{0}(L+w)\left(\alpha_{2 z}+v_{1 z}\right) \frac{d w}{v} d v d u-\int_{0}^{t} \int_{0}^{\varphi}(\varphi-v) \int_{-L}^{0}(L+w) \times\right. \\
& \times\left(\alpha_{2 z}+v_{1 z}\right) d w d v d \tau+\int_{0}^{t} \int_{0}^{r}(r-u) \int_{0}^{\varphi}(\varphi-v) \int_{-L}^{0}(L+w)\left(\alpha_{2 z}+v_{1 z}\right) \frac{\partial v}{\partial \tau} \frac{d w}{v^{2}} d v d u d \tau+ \\
& +\frac{1}{2} \int_{0}^{t} \int_{0}^{\varphi}(\varphi-v) \int_{-L}^{0}(L+w) \frac{v_{r}^{2}}{v} d w d v d \tau+\frac{1}{2} \int_{0}^{t} \int_{0}^{r}(r-u) \int_{0}^{\varphi}(\varphi-v) \int_{-L}^{0}(L+w)\left(\alpha_{2 r}+v_{1 r}\right)^{2} \frac{\partial v}{\partial u} \times \\
& \times \frac{d w}{v^{2}} d v d u d \tau+\int_{0}^{t} \int_{0}^{r} \frac{(r-u)}{2 u} \int_{0}^{\varphi} \int_{-L}^{0}(L+w)\left(\alpha_{2 \varphi}+v_{1 \varphi}\right)^{2} \frac{d w}{v} d v d u d \tau+\frac{1}{2} \int_{0}^{t} \int_{0}^{r}(r-u) \times \\
& \times \int_{0}^{\varphi}(\varphi-v) \int_{-L}^{0}\left(\alpha_{2 z}+v_{1 z}\right)^{2} \frac{d w}{v} d v d u d \tau-\int_{0}^{t} \int_{0}^{r} \frac{(r-u)}{u^{2}} \int_{-L}^{0}(L+w)\left(\alpha_{2 z}+v_{1 z}\right) d w d u d \tau+ \\
& +\frac{1}{2} \int_{0}^{t} \int_{0}^{r}(r-u) \int_{0}^{\varphi}(\varphi-v) \int_{-L}^{0} w\left(\alpha_{2 z}+v_{1 z}\right)^{2} \frac{\partial v}{\partial w} \frac{d w}{v^{2}} d v d u d \tau+\frac{L}{2} \int_{0}^{t} \int_{0}^{r}(r-u) \int_{0}^{\varphi}(\varphi-v) \int_{-L}^{0}\left(\alpha_{2 z}+\right. \\
& \left.\left.+v_{1 z}\right)^{2} \frac{\partial v}{\partial w} \frac{d w}{v^{2}} d v d u d \tau+\frac{1}{2} \int_{0}^{t} \int_{0}^{r} \frac{r-u}{u} \int_{0}^{\varphi} \int_{-L}^{0}(L+w)\left(\alpha_{2 \varphi}+v_{1 \varphi}\right)^{2} \frac{\partial v}{\partial v} \frac{d w}{v^{2}} d v d u d \tau\right]- \\
& \left.-\int_{0}^{t} \int_{0}^{r}(r-u) \int_{0}^{\varphi}(\varphi-v) \frac{P}{\rho} d v d u d \tau\right\}
\end{aligned}
$$

Values of parameters $A_{i}, B_{i}$ and $C_{i}$ could be determined by the following relations

$$
\begin{aligned}
& A_{1}=\frac{R^{2}}{8} \mu_{012 r 12}-\frac{R}{4} \mu_{112 r 12}-\frac{1}{4} \mu_{112 r 01}-\frac{1}{8} \mu_{212 r 12}+\frac{R}{4} \mu_{012 r 01}-\frac{L}{8} \widetilde{\mu}_{211 r 12}+L \frac{R^{2}}{8} \widetilde{\mu}_{011 r 01}+L \frac{R}{4} \times \\
& \times \widetilde{\mu}_{011 r 01}+L \frac{R}{4} \widetilde{\mu}_{011 r 01}-L \frac{R}{4} \widetilde{\mu}_{111 r 12} \quad A_{2}=\frac{R^{2}}{4} \mu_{011 \varphi 12}-\frac{1}{4} \mu_{212 \varphi 12}-\frac{R}{4} \mu_{012 \varphi 12}-\frac{L}{8} \widetilde{\mu}_{211 \varphi 12}-
\end{aligned}
$$


$-L \frac{R}{4} \widetilde{\mu}_{011 \varphi 12}+L \frac{R^{2}}{8} \widetilde{\mu}_{010 \varphi 12}+\frac{R}{2} \widetilde{\mu}_{011 \varphi 12} \quad A_{3}=\frac{R}{4} \mu_{012 z 12}-\frac{1}{4} \mu_{212 z 12}-\frac{R}{2} \mu_{011 z 01}+\widetilde{\mu}_{010 z 12} \times$ $\times L \frac{R}{4}-\frac{R}{2} \mu_{011 z 12}-\frac{R^{2}}{4} \widetilde{\mu}_{011 z 12}-\frac{R}{2} \widetilde{\mu}_{010 z 01} ; \quad A_{4}=\frac{R^{2}}{4} \Omega_{0121 r r 121}-\frac{R}{2} \mu_{112 r 01}-\frac{R}{2} \mu_{112 t 12}-\frac{R}{2} \times$ $\times \Omega_{1121 r r 121}-\frac{1}{8} \mu_{222 r 01}-\frac{1}{8} \mu_{212 r 01}-\frac{1}{4} \mu_{212 t 10}-\frac{1}{2} \Omega_{212 r r 011}+\frac{R^{2}}{4} \mu_{012 t 12}-\frac{1}{2} \Omega_{212 r r 1121}-\widetilde{\mu}_{211 \kappa 12} \times$ $\times \frac{L}{8}-\Theta^{2} L R^{3} \frac{\pi}{3}+\frac{R}{2} \Omega_{012 r r 1011}+\Theta^{2} R^{2} L^{2} \frac{\pi^{2}}{2}-\frac{L}{4} \widetilde{\mu}_{211 r 01}-\frac{L}{4} \widetilde{\mu}_{211 t 12}-\frac{L}{4} \widetilde{\Omega}_{211 r r 1121}-\frac{7}{2} \pi^{2} \Theta^{2} \times$ $\times R^{3} L-\frac{L}{4} \widetilde{\mu}_{211 r 01}+L \frac{R^{2}}{4} \widetilde{\mu}_{011 t 12}+L \frac{R^{2}}{4} \widetilde{\Omega}_{011 r r 1121}+L \frac{R^{2}}{4} \widetilde{\mu}_{011 r 01}+L \frac{R}{2} \widetilde{\Omega}_{011 r r 1011}-R \frac{L}{2} \widetilde{\mu}_{111 r 01}+$ $+R \frac{L}{2} \widetilde{\Omega}_{011 r r 1011}-L \frac{R}{2} \widetilde{\Omega}_{111 r r 1121} \Omega_{i j k \rho l m n o}=\int_{0}^{\Theta}(\Theta-t) \int_{0}^{R} \int_{0}^{2 \pi}(2 \pi-\varphi)^{j} \int_{-L}^{L}(L-z)^{k}\left(v_{l \rho}\right)^{o}\left(\frac{\partial v}{\partial \lambda}\right)^{m} \frac{d z}{v^{n}} d \varphi \times$ $\times(R-r)^{i} d r d t ; \quad \widetilde{\Omega}_{i j k \rho l m n o}=\int_{0}^{\Theta}(\Theta-t) \int_{0}^{R}(R-r)^{i} \int_{0}^{2 \pi}(2 \pi-\varphi)^{j} \int_{-L}^{0}(L-z)^{k}\left(v_{l \rho}\right)^{o}\left(\frac{\partial v}{\partial \lambda}\right)^{m} \frac{d z}{v^{n}} d \varphi d r d t$ $A_{5}=\frac{R^{2}}{2} \Omega_{012 \varphi \varphi 1121}-\frac{1}{4} \Omega_{212 \varphi \varphi 1121}-\frac{R}{2} \Omega_{012 \varphi \varphi 1121}-\frac{L}{4} \Omega_{211 \varphi \varphi 1121}-R \frac{L}{2} \Omega_{011 \varphi \varphi 1121}+\Omega_{010 \varphi \varphi 1121} \times$ $\times L \frac{R^{2}}{4}-\frac{L}{2} R \widetilde{\mu}_{111 t 12}+R \widetilde{\Omega}_{011 \varphi \varphi 1121} ; \quad A_{6}=\frac{R^{2}}{4} \Omega_{012 z 1121}-\frac{1}{4} \Omega_{212 z 1121}-\frac{1}{4} \mu_{210 z 0}-\Omega_{010 z 1011} \times$ $\times R+\pi^{2} \Theta^{2} R^{2} L^{2}+L \frac{R^{2}}{4} \widetilde{\Omega}_{011 z 1121}-R \widetilde{\Omega}_{011 z 1121}+R \frac{L}{2} \widetilde{\Omega}_{010 z 1011}+L \frac{R}{2} \widetilde{\Omega}_{010 z 1121} ; A_{7}=$ $=\frac{1}{2} \Omega_{210 r r 1001}--\frac{1}{2} \Omega_{121 r r 1001}-\frac{1}{4} \Omega_{212 t r 101}-\Omega_{221 z z 1001}+\frac{1}{4} \Omega_{212 r r 1011}+\frac{1}{4} \Omega_{212 r r 1012}+\frac{1}{4} \Omega_{211 z z 1011}-$ $-\frac{1}{8} \Omega_{212 r r 2122}-\frac{1}{8} \Omega_{212 \varphi \varphi 2122}-R \Omega_{110 r r 1001}-\frac{1}{8} \Omega_{212 z z 2122}+\frac{1}{2} \Omega_{112 r r 1011}-R \Omega_{011 r r 1001}+\Omega_{012 r r 1122} \times$ $\times \frac{R^{2}}{8}-\frac{R^{2}}{4} \Omega_{012 r t 1121}+R \Omega_{110 r r 1001}+R \Omega_{011 z z 1001}+\frac{R}{2} \Omega_{112 r r 1011}-\frac{R^{2}}{8} \Omega_{012 z z 1122}+\frac{R}{4} \Omega_{112 r t 1121}+$ $+\frac{R}{4} \Omega_{112 r r 122}-\frac{R^{2}}{4} \Omega_{0121 \varphi \varphi 1122}+\frac{R}{4} \Omega_{012 \varphi \varphi 1122}+\frac{R}{2} \Omega_{011 z z 1012}-\frac{R}{4} \Omega_{012 r r 1012}-\frac{R}{4} \Omega_{012 r r 1012}+\frac{R}{2} \times$ $\times \Omega_{011 z z 1122}+R^{2} L \Omega_{010 r r 1001}-\frac{L}{4} \widetilde{\Omega}_{211 r r 1011}-\frac{L}{4} \widetilde{\Omega}_{211 r r 121}-\frac{L}{4} \widetilde{\Omega}_{110 r r 1001}-\frac{L}{8} \widetilde{\Omega}_{211 r r 122}-\frac{L}{2} \times$ 


$$
\begin{aligned}
& \times \widetilde{\Omega}_{110 z z 1001}-\frac{L}{4} \widetilde{\Omega}_{111 r r 1011}-\frac{L}{2} \widetilde{\Omega}_{211 \varphi \varphi 1122}+V_{0}^{2} L \frac{R}{4} \widetilde{\mu}_{010 r 01}-L \frac{R}{4} \widetilde{\Omega}_{011 \varphi \varphi 1122}+L \widetilde{\Omega}_{011 r t 1121} \frac{R^{2}}{4}+ \\
& +L \frac{R^{2}}{8} \widetilde{\Omega}_{011 r r 1122}+L \frac{R^{2}}{8} \widetilde{\Omega}_{010 \varphi \varphi 1122}+L \frac{R^{2}}{4} \widetilde{\Omega}_{011 r r 1011}+\frac{L}{2} \widetilde{\Omega}_{011 r r 1001}+L \frac{R}{4} \widetilde{\Omega}_{011 r 11012}-R \frac{L}{2} \times \\
& \times \widetilde{\Omega}_{111 r r 1011}+R \frac{L}{2} \widetilde{\Omega}_{010 r r 1001}+L \frac{R}{4} \widetilde{\Omega}_{011 r r 1012}+L \frac{R^{2}}{8} \widetilde{\Omega}_{011 z z 1122}-R \frac{L}{2} \widetilde{\Omega}_{111 r t 1121}+R \frac{L}{2} \widetilde{\Omega}_{010 z 1001}+ \\
& +L \frac{R}{4} \widetilde{\Omega}_{010 z z 1012}-L \frac{R}{4} \widetilde{\Omega}_{011 \varphi \varphi 1121}+L \frac{R}{4} \widetilde{\Omega}_{010 z z 1122}-L \frac{R}{4} \widetilde{\Omega}_{111 r r 1122}-L \frac{R}{2} \int_{0}^{\Theta}(\Theta-t) \int_{0}^{2 \pi}(2 \pi-\varphi) \times \\
& \times \int_{-L}^{0} \frac{P}{\rho} d z d \varphi d t+\int_{0}^{\Theta}(\Theta-t) \int_{0}^{R}(R-r) \int_{0}^{2 \pi}(2 \pi-\varphi) \int_{-L}^{L}(L-z) \frac{P}{\rho} d z d \varphi d u d t \quad B_{1}=\frac{1}{8} \mu_{222 r 01}- \\
& -\frac{1}{2} \mu_{322 r 01}+\frac{1}{4} \mu_{322 r 12}-\frac{\pi}{4} \mu_{212 r 01}+\frac{\pi}{2} \mu_{312 r 01}-\frac{\pi}{4} \mu_{312 r 12}-\frac{L}{8} \mu_{221 r 01}-\frac{L}{8} \hat{\mu}_{321 r 12}+\frac{\pi^{2}}{8} \times \\
& \times \Theta^{2} R^{2} L^{3}-\hat{\mu}_{321 r 01} \frac{L}{4}+L \frac{\pi}{4} \hat{\mu}_{211 r 01}+L \frac{\pi}{4} \hat{\mu}_{311 r 12}-\pi \frac{L}{2} \hat{\mu}_{211 r 01} \quad B_{2}=\frac{1}{2} \mu_{222 r 01}+L \frac{\pi}{4} \times \\
& \times \hat{\mu}_{201 r 01}-\frac{\pi}{2} \mu_{202 r 01}-\frac{L}{4} \hat{\mu}_{i j k \rho l m} \mu_{211 r 01} B_{3}=\frac{1}{4} \mu_{321 r 01}+\frac{1}{8} \mu_{322 z 12}-\frac{\pi}{2} \mu_{311 z 01}-\frac{\pi}{4} \mu_{312 z 12}- \\
& -\frac{L}{8} \mu_{121 z 12}-\frac{L}{8} \mu_{320 z 01}+L \frac{\pi}{4}-\frac{\pi}{4} \mu_{310 z 01}+L \frac{\pi}{4}-\frac{\pi}{4} \mu_{310 z 12} ; \quad B_{4}=\frac{1}{4} \Omega_{202 r r 1011}-\Theta^{2} R^{2} L^{2} \times \\
& \times \frac{\pi^{2}}{4}--\frac{1}{2} \Omega_{322 r r 1011}+\frac{1}{4} \Omega_{322 r r 1121}-\frac{\pi}{2} \Omega_{212 r r 1011}+\pi \Omega_{312 r r 1011}-\frac{\pi}{2} \Omega_{312 r r 1121}-\frac{L}{4} \Omega_{221 r r 1011}- \\
& -\frac{L}{4} \hat{\Omega}_{321 r r 1121}-\frac{L}{2} \hat{\Omega}_{321 r r 1011}+\pi \frac{L}{2} \hat{\mu}_{211 r 01}-\pi L \hat{\Omega}_{101 r r 1001}+\pi \frac{L}{2} \hat{\Omega}_{311 r r 1121}+\pi L \hat{\Omega}_{211 r r 1011} \text {; } \\
& B_{5}=\frac{1}{4} \mu_{322 r 01}+\frac{1}{4} \mu_{322 t 12}+\frac{1}{4} \mu_{322 t 12}+\frac{1}{2} \Omega_{212 \varphi \varphi 1011}-\frac{\pi}{2} \mu_{312 r 01}-\frac{\pi}{2} \Omega_{202 \varphi \varphi 1011}-\frac{1}{2} \mu_{312 t 12}+\Theta^{2} \frac{\pi^{3}}{3} R^{3} L- \\
& -\frac{L}{4} \mu_{321 t 01}-\frac{L}{4} \hat{\mu}_{321 r 01}-\frac{L}{4} \hat{\mu}_{321 t 12}-\frac{L}{2} \hat{\mu}_{211 \varphi 01}+L \frac{\pi}{2} \hat{\mu}_{211 t 12}+L \frac{\pi}{2} \hat{\mu}_{311 r 01}+L \frac{\pi}{2} \hat{\mu}_{201 \varphi 01 ;} B_{6}= \\
& =\frac{1}{2} \Omega_{321 z z 1011}+\frac{1}{4} \Omega_{322 z z 1121}-\pi \Omega_{311 z z 1011}-\pi \Omega_{312 z z 121}-\frac{L}{4} \Omega_{321 z z 1011}-\frac{L}{4} \hat{\Omega}_{321 z z 1121}-\hat{\Omega}_{321 z z 1121} \times \\
& \times \pi \frac{L}{2}-\pi \frac{L}{2} \hat{\Omega}_{310 z z 1011} ; \quad B_{7}=\frac{1}{2} \Omega_{112 r r 1001}-\Omega_{212 r r 1001}-\Omega_{111 r r 1001}+\frac{1}{2} \Omega_{220 \varphi \varphi 1001}- \\
& -\frac{1}{4} \Omega_{322 \varphi \varphi 1011}-\frac{1}{8} \Omega_{222 r r 1012}-\frac{1}{2} \Omega_{322 \varphi t 1121}-\frac{1}{8} \Omega_{322 r r 1122}+\frac{1}{4} \Omega_{322 r r 1012}-\frac{1}{4} \Omega_{321 z z 1012}-
\end{aligned}
$$




$$
\begin{aligned}
& -\frac{1}{4} \Omega_{212 \varphi \varphi 1012}-\frac{1}{8} \Omega_{322 z z 1122}+\frac{\pi}{2} \Omega_{002 r r 1001}+\pi \Omega_{101 r r 1001}-\frac{\pi}{2} \Omega_{102 r r 1001}+\frac{\pi}{4} \Omega_{212 r r 1012}+ \\
& +\frac{\pi}{2} \Omega_{312 \varphi \varphi 1011}+\frac{\pi}{2} \Omega_{312 \varphi t 1121}-\pi \Omega_{210 \varphi \varphi 1001}-\frac{\pi}{2} \Omega_{312 r r 1012}+\frac{\pi}{4} \Omega_{312 r r 1122}+\frac{\pi}{2} \Omega_{311 z z 1012}+ \\
& +\frac{\pi}{4} \Omega_{312 z z 1122}-\frac{L}{2} \Omega_{111 r r 1001}+\frac{L}{2} \Omega_{111 r r 1001}+\frac{L}{8} \Omega_{221 r r 1012}+\frac{L}{8} \hat{\Omega}_{321 r r 1122}+\frac{L}{4} \hat{\Omega}_{321 \varphi \varphi 1011}+\frac{L}{4} \times \\
& \times \hat{\Omega}_{321 \varphi \varphi 1011}+\frac{L}{4} \hat{\Omega}_{321 \varphi t 1121}-\omega^{2} L R^{5} \Theta^{2} \frac{\pi^{2}}{20}+\frac{L}{4} \hat{\Omega}_{321 r r 1012}+\frac{L}{4} \hat{\Omega}_{312 z 1012}+\frac{L}{2} \hat{\Omega}_{110 r r 1001}+ \\
& +\frac{L}{8} \hat{\Omega}_{321 z z 1122}+\frac{L}{4} \hat{\Omega}_{211 \varphi \varphi 1012}+L \frac{\pi}{2} \hat{\Omega}_{101 r r 1002}-L \frac{\pi}{4} \hat{\Omega}_{011 r r 2012}-L \frac{\pi}{2} \hat{\Omega}_{101 r r 1011}-\hat{\Omega}_{100 r r 1001} \times \\
& \times \frac{\pi}{2} L-L \frac{\pi}{2} \hat{\Omega}_{311 \varphi t 1121}-\pi \frac{L}{2} \hat{\Omega}_{311 \varphi \varphi 1011}-L \frac{\pi}{4} \hat{\Omega}_{311 r r 1122}+\pi \frac{L}{2} \hat{\Omega}_{311 r r 1012}-L \frac{\pi}{4} \hat{\Omega}_{310 z z 1012}- \\
& -L \frac{\pi}{4} \hat{\Omega}_{310 z z 1122}-L \frac{\pi}{4} \hat{\Omega}_{201 \varphi \varphi 1012}-\int_{0}^{\Theta}(\Theta-t) \int_{0}^{R}(R-r) \int_{0}^{2 \pi}(2 \pi-\varphi) \int_{-L}^{L}(L-z) \frac{P}{\rho} d z d \varphi d r d t+ \\
& +\omega^{2} R^{5} L \Theta^{2} \frac{\pi^{2}}{40} ; \quad C_{1}=-\frac{1}{16} \mu_{222 r 12}-\frac{1}{16} \mu_{222 r 01}-\frac{L}{8} \widetilde{\mu}_{021 r 01}-\frac{L}{16} \widetilde{\mu}_{221 r 12} \quad C_{2}=\frac{1}{8} \mu_{122 \varphi 12}+ \\
& +\frac{1}{4} \mu_{122 z 01}-\frac{L}{16} \widetilde{\mu}_{221 z 01}-\frac{L}{16} \widetilde{\mu}_{221 z 12}-\frac{L}{8} \widetilde{\mu}_{211 z \varphi 1}-\frac{L}{8} \widetilde{\mu}_{221 \varphi 12} \quad C_{3}=-\frac{1}{16} \mu_{222 z 12}-\frac{1}{16} \mu_{222 z 01}- \\
& -\frac{L^{2}}{18} \widetilde{\mu}_{220 z 12} \quad C_{4}=2 L \Theta^{2} R^{2} \frac{\pi^{3}}{9}-\frac{1}{16} \Omega_{222 r r 121}-\frac{1}{8} \Omega_{222 r r 1011}-\frac{L}{16} \widetilde{\mu}_{221 r r 1121}-\frac{L}{4} \widetilde{\Omega}_{021 r r 1011} \text {; } \\
& C_{5}=-\frac{1}{4} \Omega_{122 \varphi \varphi 1121}-\frac{1}{4} \Omega_{222 \varphi \varphi 1011}+\frac{L}{4} \widetilde{\Omega}_{211 \varphi \varphi 1011}-\frac{L}{4} \widetilde{\Omega}_{211 \varphi \varphi 1121} ; \quad C_{6}=17 R \Theta^{2} L^{3} \frac{\pi^{3}}{9}- \\
& -\frac{1}{8} \mu_{222 t 12}-\frac{1}{8} \Omega_{222 z z 1121}-\frac{1}{8} \mu_{222 t 01}-\frac{1}{8} \Omega_{222 z z 1011}-\frac{L}{8} \widetilde{\mu}_{221 t 01}-\frac{L}{8} \widetilde{\mu}_{221 t 12}-\frac{L}{8} \widetilde{\mu}_{220 z 01}+\pi L^{3} \times \\
& \times R^{3} \frac{\Theta^{2}}{48}-\frac{L}{8} \widetilde{\Omega}_{221 z z 1121}-\frac{L^{2}}{8} \widetilde{\Omega}_{220 z z 1121} \quad C_{7}=\frac{1}{16} \Omega_{222 r r 1122}+\frac{1}{16} \Omega_{222 r r 1012}+\frac{1}{4} \Omega_{221 r r 1001}+ \\
& +\frac{1}{4} \Omega_{022 z z 1001}+\frac{1}{4} \Omega_{212 z z 1001}+\frac{1}{4} \Omega_{122 z t 1121}+\frac{1}{8} \Omega_{122 \varphi \varphi 1122}+\frac{1}{16} \Omega_{222 z z 1122}+\frac{1}{8} \Omega_{122 z z 1011}+\frac{1}{16} \times \\
& \times \Omega_{222 z z 1012}+\frac{1}{8} \Omega_{122 \varphi \varphi 1012}+\frac{1}{4} \widetilde{\Omega}_{221 z z 1011}-\frac{L}{4} \widetilde{\Omega}_{021 z z 1001}+\frac{L}{8} \widetilde{\Omega}_{221 z t 1121}+\frac{L}{8} \widetilde{\Omega}_{021 r r 1012}+\frac{L}{16} \times \\
& \times \widetilde{\Omega}_{221 r r 1122}+\frac{L}{8} \widetilde{\Omega}_{211 \varphi \varphi 1012}+\frac{L}{16} \widetilde{\Omega}_{220 z z 1012}-\frac{L}{4} \widetilde{\Omega}_{201 z z 1001}+\frac{L}{16} \widetilde{\Omega}_{221 z z 1122}+\frac{L^{2}}{16} \widetilde{\Omega}_{220 z z 1122}-
\end{aligned}
$$




$$
-\frac{L}{8} \widetilde{\Omega}_{212 \varphi \varphi 1122}+\frac{1}{2} \int_{0}^{\Theta}(\Theta-t) \int_{0}^{R}(R-r)^{2} \int_{0}^{2 \pi}(2 \pi-\varphi)^{2} \int_{-L: \rho}^{L} \frac{P}{\rho} d z d \varphi d r d t
$$

\section{REFERENCES}

[1] I.P. Stepanenko. Basis of microelectronics. Moscow, Soviet radio (in Russian), 1980.

[2] V.G. Gusev, Yu.M. Gusev. Electronics. Moscow, Higher School (in Russian), 1991.

[3] V.I. Lachin, N.S. Savelov. Electronics. Rostov-on-Don, Phenics, 2001.

[4] A.A. Vorob'ev, V.V. Korabl'ev, S.Yu. Karpov. Semiconductors. Vol. 37 (7). P. 98 (2003).

[5] L.M. Sorokin, N.V. Veselov, M.P. Shcheglov, A.E. Kalmykov, A.A. Sitnikova, N.A. Feoktistov, A.V. Osipov, S.A. Kukushkin. Technical Physics Letters. Vol. 34 (22). P. 68 (2008).

[6] V.V. Lundin, A.V. Sakharov, E.E. Zavarin, M.A. Sinitsin, A.E. Nikolaevb, G.A. Mikhailovsky, P.N. Brunkov, V.V. Goncharov, B.Ya. Ber, D.Yu. Kazantsev, A.F. Tsatsul'nikov. Semiconductors. Vol. 43 (7). P. 996 (2009).

[7] Y. Li, L.E. Antonuk, Y. El-Mohri, Q. Zhao, H. Du, A. Sawant, Yi Wang. J. Appl. Phys. Vol. 99 (6). P. 064501 (2006).

[8] Chakraborty, H. Xing, M.D. Craven, S. Keller, T. Mates, J. S. Speck, S.P. Den Baars, U.K. Mishra. J. Appl. Phys. Vol. 96 (8). P. 4494 (2004).

[9] M. Mitsuhara, M. Ogasawara, H. Sugiura. J. Cryst. Growth. Vol. 183. P. 38 (1998).

[10] R.A. Talalaev, E.V. Yakovleva, S.Yu. Karpova, Yu.N. Makarov. J. Cryst. Growth. Vol. 230. P. 232 (2001).

[11] H.S. Carslaw, J.C. Jaeger. Conduction of heat in solids. - Oxford: At the Clarendon Press. 1964. 488 p.

[12] Yu.D. Sokolov. Applied Mechanics. Vol.1 (1). P. 23-35 (1955).

[13] E.L. Pankratov. J. Comp. Theor. Nanoscience. Vol.9 (1). P. 41-49 (2012).

[14] E.L. Pankratov, E.A. Bulaeva. Nanoscience and Nanotechnology Letters. Vol. 5 (3). P. $418-426$ (2013).

[15] E.L. Pankratov, E.A. Bulaeva. J. Adv. Phys. Vol. 2 (1). P. 75-84 (2013).

[16] G. Korn, T. Korn. Mathematical Handbook for scientists and engineers. Definitions, theorems and formulas for reference and review. Second edition. McGraw-Hill Book Company. New York, 1968.

[17] E.L. Pankratov, E.A. Bulaeva. Reviews in Theoretical Science. Vol. 1 (3). P. 305-316 (2013). 